\title{
Stochastic Deconvolution Over Groups
}

\author{
Birsen Yazici, Member, IEEE
}

\begin{abstract}
In this paper, we address a class of inverse problems that are formulated as group convolutions. This is a rich area of research with applications to Radon transform inversion for tomography, wide-band and narrow-band signal processing, inverse rendering in computer graphics, and channel estimation in communications, as well as robotics and polymer science. We present a group-theoretic framework for signal modeling and analysis for such problems and propose a minimum mean-square error (MMSE) deconvolution method in a probabilistic setting. Key components of our approach are group representation theory and the concept of group stationarity. The roposed deconvolution method incorporates a priori information and noise statistics into the inversion process, which leads to a natural regularized solution. We present recovery of self-similar processes that are "blurred" and embedded in noise as a demonstration example. The method is applicable to a wide range of inverse problems involving both commutative and noncommutative groups including finite, compact, and majority of well-behaved locally compact groups.
\end{abstract}

Index Terms-Deconvolution, group convolution, group representation theory, inverse problems, minimum mean-square error (MMSE) filtering, Wiener filtering.

\section{INTRODUCTION}

\section{A. Motivations}

$\mathbf{T}$ HIS work addresses a class of inverse problems that are modeled as group convolutions. One common type of such problem is the classical deconvolution problem describing the image formation for space-invariant imaging systems. However, there are many imaging problems where classical convolution integral can not capture the imaging process. For example, in computed tomography, the imaging process, which is described by the Radon transform, can be viewed as a convolution integral over Euclidean motion groups. Similarly, in wide-band and narrow-band coherent imaging, physics of imaging leads to forward models that are defined as convolution integrals over the affine and Heisenberg groups, respectively. In computer vision, realistic rendering of computer-generated images requires estimation of illumination and bidirectional reflectance distribution function from real photographs, which can be modeled as a convolution integral over the rotation group [39]. Apart from these imaging problems, group convolutions appear in a broad array of inverse problems in science and engineering. These include channel estimation in wireless communications,

Manuscript received April 22, 2002; revised September 8, 2003.

The author is with the Jonsson Engineering Center, Electrical, Computer and Systems Engineering, Rensselaer Polytechnic Institute, Troy, NY 12180 USA (e-mail: yazici@ecse.rpi.edu).

Communicated by V. V. Veeravalli, Associate Editor for Detection and Estimation.

Digital Object Identifier 10.1109/TIT.2004.824916 workspace estimation in robotics, estimation of the structure of macromolecules in polymer science, just to mention a few [1]-[10].

In this paper, we describe a group-theoretic linear system and signal theory, in particular group stationarity stochastic processes and Fourier analysis over groups. We introduce the problem of convolution integral over groups and, demonstrate its occurrence in a broad array of engineering problems. We present a Wiener type minimum mean square error solution for the convolution integral and presented its application to recovery of self-similar signals embedded in noise.

Group convolution operation can be viewed as a representation of the input-output relationship of a linear system, which has dynamics invariant under the group composition law. As such, it is the generalization of the classical convolution integral associated with the linear time invariant systems where the underlying structure is the additive group. Classical Fourier based minimum mean square deconvolution techniques rely on the assumption of stationarity and time invariance, and utilize the Fourier transform to develop inverse filtering methods. In this work, we develop a stochastic inverse filtering technique based on the minimum mean square error criterion to solve the convolution integral equation for a class of locally compact groups of both commutative and noncommutative type. This class of groups includes finite, compact, and algebraic Lie groups, separable locally compact commutative groups, and a majority of well-behaved locally compact groups.

The key components of our study are Fourier transforms on groups, and the concept of group stationarity. Group stationarity can be thought of as a generalization of the ordinary stationarity in which the second-order statistics remain invariant under the group composition law. Such statistical structures can be easily induced from the white noise processes via linear group-invariant filtering. It was shown that the special cases of the group stationary processes, in which the underlying structures are multiplicative and affine groups, provides an engineering-oriented mathematical framework to model self-similar and multiresolution signals [19], [21]-[23].

The central fact in the analysis of group stationary processes is the existence of spectral decomposition theorems, which is facilitated by the theory of group representations. This area of mathematics deals with the generalization of the ordinary convolution and the Fourier analysis to functions on groups. That is, given a complex-valued function defined on a group, it can be decomposed in terms of collection of unitary irreducible representations of the group. The result is a collection of Fourier coefficients that exhibit some type of group invariance. This decomposition, which is referred to as the spectral analysis of functions, transforms convolution over the group to a multiplication in the Fourier domain. 
Statistical methods introduced in this paper complement a variety of group-theoretical methods that have been formulated in a deterministic setting. For example, in inverse problems involving group convolutions, Fourier domain inversion techniques do not consider noise in measurements [56]. Our deconvolution technique not only utilizes noise statistics, but also provides a possible regularization to the inversion problem. In addition to being novel, and leading to potentially improved results, the implementation of these techniques can be achieved efficiently using fast Fourier transform (FFT) algorithms [27]-[29] available for a broad range of groups.

Finally, we believe that group theory not only provides physically intuitive and computationally efficient methods, but also a mathematically unifying and powerful approach for both linear systems theory, transform theory, and statistical signal modeling and processing. This geometric connection between the system theory and statistical tools provides a deeper understanding of signal and system structures and the associated processing techniques.

\section{B. Related Work}

The particular focus of our work utilizes noncommutative harmonic analysis over groups to solve convolution integrals in a probabilistic setting. To the best of our knowledge, there is a limited number of studies in the literature on this specific topic. In [9], Naparst addressed deconvolution over the affine group in the context of wide-band target density estimation for sonar and radar applications. In [56], Kyatkin and Chirikjian developed a regularized solution for a specific convolution integral equation over the Euclidean motion group and demonstrated its application into the kinematic design of binary manipulators [5]. Both of these studies address specific problems in deterministic setting. Our work addresses the deconvolution problem in a probabilistic setting for a broad range of topological groups that arise naturally in engineering applications. Our minimum mean-square formulation also provides a natural regularization to the inverse problem. Another work that is related to our research is the work of Karpovsky and Trachtenberg [41]-[43]. In [43], they addressed the problem of nonstationary signal recovery in the presence of additive noise. The primary objective of this work is to take advantage of fast unitary transforms over finite groups to obtain suboptimal approximations to Wiener filtering achieved by Karhune-Loéve transforms. Additionally, a number of interesting studies recently appeared in the statistics literature addressing deconvolution over groups, in which the underlying functions are probability density functions; see, for example, [63] and the references therein. These studies address a very interesting but entirely different problem. In this class of deconvolution problems, the noise model is not additive but rather acts on the signal via a transitive group action. Thus, the deconvolution problem is to recover the probability density function of the unknown signal from the probability density function of the measurements.

Apart from these studies, group theory appears in pattern recognition, signal processing, and information theory in a variety of other ways. Most notably, in the areas of coding theory, fast transforms, wavelet theory, and pattern matching [7], [57], [25], [27], [28], [1]-[3], [64], [65].

\section{Organization}

In Section II, we introduce two image reconstruction problems that can be formulated and addressed within the mathematical framework that will be introduced in this paper. This section provides theoretical and engineering motivations for our development in the subsequent sections. In Section III, we introduce the concept of convolution and Fourier analysis on groups and describe group-theoretical signal processing in a system-theoretic framework. In Section IV, we introduce stochastic processes that are indexed by groups and their spectral decomposition theorems. In each of these sections, the theory is illustrated by examples. In Section V, we present minimum mean-square solution of the convolution integral. In Section VI, we demonstrate the application of the proposed inverse filtering method in the recovery of blurred and noisy self-similar signals. Finally, in Section VII, we point out future research directions and conclude our discussion.

\section{IMAGE RECONSTRUCTION APPLICATIONS}

Before we start our discussion, we will give an overview of two application areas where our results are directly applicable. Both of these problems can be viewed as inverse imaging problems.

\section{A. Radar and Sonar Image Reconstruction by Wide-Band and Narrow-Band Processing}

In radar and sonar imaging, the transmitter emits an electromagnetic signal. The signal is reflected off a target and detected by the transmitter/receiver as an echo signal. Assuming negligible acceleration of the reflector, the wide-band model of the echo from a point reflector is given as the time-delayed and time-scaled replica of the transmitted pulse [13]-[15]:

$$
e(t)=\sqrt{s} f(s t+\tau)
$$

where $f$ is the transmitted pulse, $\tau$ is the time delay, and $s$ is the time scale or Doppler stretch. The term $\sqrt{s}$ is needed, if we require the energy of the echo signal is to be conserved. It is given as $s=(c-v) /(c+v)$, where $c$ is the speed of the transmitted signal propagating in a homogenous medium and $v$ is the radial velocity of the reflector. Wide-band processing applies to several types of signals, including signals with large fractional bandwidths, signals with large time bandwidths, signals whose statistics may be nonstationary in the ordinary sense, and signals reflected from rapidly moving objects. The advantage of wide-band processing includes noise immunity, improved resolution, and removal of many assumptions. Wide-band processing has been studied for decades. However, its implementation has been limited since wide-band techniques could not be efficiently implemented.

The narrow-band model of the echo from a point reflector is given by

$$
e(t)=f(t-\tau) e^{j \omega t}
$$

where $f$ is the transmitted pulse, $\tau$ is the time delay, and $\omega$ is the frequency or Doppler shift. When $|v|<<c$ and $t$ is small over 
a period of observations, the wide-band model can be approximated by the narrow-band model. (See [30] for a careful analysis of wide-band solution and narrow-band approximation.) Generally speaking, the narrow-band approximation is usually adequate for radar applications but is less appropriate for sonar.

It is often desirable to image a dense group of reflectors, which may be several objects or a single object distributed in size. This dense group of reflectors is then described by a reflectivity density function. The received signal is modeled as a weighted average [7]-[9]. For wide-band signals, it is given as

$$
e_{W}(t)=\int_{-\infty}^{\infty} \int_{0}^{\infty} S_{W}(s, \tau) \frac{1}{\sqrt{s}} f\left(\frac{t-\tau}{s}\right) \frac{d s}{s^{2}} d \tau
$$

where $S_{W}(s, \tau)$ is the wide-band reflectivity density function associated with each time-delayed and time-scaled version of the transmitted signal. The narrow-band model is given by

$$
e_{N}(t)=\int_{-\infty}^{\infty} \int_{-\infty}^{\infty} S_{N}(\omega, \tau) f(t-\tau) e^{j \omega t} d \tau d \omega
$$

where $S_{N}(\omega, \tau)$ is the narrow-band reflectivity density function associated with each time-delayed and frequency-shifted version of the transmitted signal.

The goal in radar and sonar imaging is to estimate $S_{W}(s, \tau)$ and $S_{N}(\omega, \tau)$ given the transmitted and the received signals. Typically, the received echo in a radar or sonar system is very weak due to clutter and system noise. Therefore, the detection at the receiver side is performed by matched filtering, which amounts to correlating the received echo with the transmitted pulse. When the two echo models described in (2.3a) and (2.3b) are inserted into the narrow-band and wide-band correlation receivers, the resulting outputs are expressed as group convolution integrals. (See [15] for a description of estimator/correlator receiver.) In the case of wide-band processing, the estimator/correlator output $C_{e f}^{W}$ is given by

$$
C_{e f}^{W}(s, \tau)=\int_{-\infty}^{\infty} \int_{0}^{\infty} S_{W}(a, b) A_{W}\left(\frac{s}{a}, \frac{\tau-b}{a}\right) \frac{d a}{a^{2}} d b
$$

where $A_{W}$ is the wide-band autoambiguity function of the transmitted pulse. Clearly, the output is expressed as a convolution integral over the affine group

$$
C_{e f}^{W}=S_{W} * A_{W} .
$$

Similarly, for the narrow-band case, the estimator/correlator output is given by

$$
\begin{array}{r}
C_{e f}^{N}(\omega, \tau)=\int_{-\infty}^{\infty} \int_{-\infty}^{\infty} S_{N}(v, \zeta) A_{N}(\omega-v, \tau-\zeta) \\
\cdot e^{-j \zeta(\omega-v)} d v d \zeta
\end{array}
$$

where $A_{N}$ is the narrow-band autoambiguity function. Thus, the output $C_{e f}^{N}$ is expressed as a convolution integral over the Heisenberg group

$$
C_{e f}^{N}=S_{N} * A_{N} .
$$

We will show that the deconvolution techniques described here provides a minimum mean-square estimate of the target re- flectivity density functions embedded in noise. For a group-theoretic approach to radar signal processing, the reader is referred to a recent article by Moran [80], and the the works of Schempp, Miller, and Naparts [8], [9], [78], [79].

\section{B. Radon Transform Inversion for Tomographic Image Reconstruction}

The Radon transform and its generalizations arise in diverse engineering applications, including medical imaging, synthetic-aperture radar (SAR), radio astronomy, and pattern recognition [12]. The Radon transform plays an important role in image reconstruction problems because it constitutes a good model of tomographic acquisition process for X-ray computed tomography (CT), single photon emission computer tomography (SPECT), positron emission tomography (PET), and SAR [31]. The problem of image reconstruction is equivalent to computing the inverse Radon transform. For recent studies, see [32]-[38]. Here, we will show that tomographic image reconstruction can be posed as a deconvolution problem over the Euclidean motion groups.

In X-ray CT, an X-ray beam with known energy is sent through the object and the attenuated X-ray is collected by an array of collimated detectors. The attenuation in the final $\mathrm{X}$-ray beam provides a means of determining the integral of the mass density of the object along the path of the X-ray. In two dimensions (2D), the relationship between the mass density along the path and the attenuation at angle $\theta$ and radius $t$ is given by the following Radon transform:

$$
p(t, \theta)=\int_{-\infty}^{\infty} \int_{-\infty}^{\infty} f(x, y) \delta(t-x \cos \theta-y \sin \theta) d x d y
$$

where $\delta$ is the Dirac delta function. Similarly, in PET and SPECT, the line projections and the attenuation coefficients are related by the Radon transform.

The Euclidean motion group is the semidirect product of the rotation group $S O(N)$ and the additive group in $\mathbb{R}^{N}$. Redefining, $\tilde{f}(R, r)=f(r) \delta(R)$, where $R$ is the two-dimensional rotation matrix, $r=[x, y]^{T}$, and $k(Q, \tau)=\delta(e \cdot \tau), e$ is the unit vector in the $x$ direction. It was observed that the Radon transforms and its generalizations can be written as a Euclidean group convolution in the following form [10], [20]:

$$
\begin{aligned}
p(R, r) & =\int_{S E(2)} k\left(g \circ h^{-1}\right) \tilde{f}(h) d h \\
& =\int_{S E(2)} k\left(R Q^{-1}, r-R Q^{-1} \tau\right) \tilde{f}(Q, \tau) d(Q, \tau) \\
g & =(R, r) \text { and } g, h \in S E(2)
\end{aligned}
$$

where $S E(2)$ denotes the two-dimensional Euclidean motion group, o denotes the group composition law, and $d(Q, \tau)$ denotes the invariant measure on the Euclidean motion group. Thus, the problem of image reconstruction is formulated as a deconvolution problem over the Euclidean motion group. By lifting the formulation of the Radon transform to the group from the coset space, one may like to take advantage of the invariance property of the group convolutions in forward and inverse calculations of the Radon transform. For example, if the object 
is translated in space by $g \in S E(2)$, its Radon transform will be translated by $g$. Therefore, the new Radon transform can be easily calculated from the original one. Similarly, in the inversion process, this property may be useful in performing spatial averaging to eliminate motion artifacts in moving objects, such as in cardiac CT imaging.

\section{THE CONCEPT OF CONVOLUTION AND FOURIER ANALYSIS ON GROUPS}

The concept of group convolution of functions on a wide variety of abstract groups is well known in pure mathematics literature [40]. Although many of the engineering applications involving group convolutions were developed independently, they can be systematically formulated within a group-invariant system-theoretic framework [21], [41], [64], [65]. Such a formulation not only establishes a relationship between classical signal and system theory, it also highlights the underlying dynamics of the group convolution filtering. Here, we shall give an overview of this topic from a perspective relevant to linear systems and signals. We start with an introduction to the concept of convolution in Section III-A, and in the following subsections, we provide a brief review of the Fourier analysis on groups.

Fourier analysis over groups allows spectral analysis of signals and systems in invariant subspaces defined by the irreducible unitary representations of the underlying group structure. This decomposition maps group convolutions into multiplications in the transform domain. Fast implementation of Fourier analysis on compact groups can be accomplished efficiently using algorithms that are analogous to the Cooley-Tukey FFT [25], [27], [28].

We shall indicate a group by $G$ and its elements by $g, h, \ldots$. The group composition law will be written by $g \circ h$, and we shall use $e$ for the identity element, for which $e \circ g=g \circ e=g$ for all elements $g$ of $G$. We shall indicate inverse elements by $g^{-1}$ so that $g^{-1} \circ g=g \circ g^{-1}=e$ for all elements $g$ of $G$. It is assumed that the reader is familiar with the concepts of group and topological spaces. Readers unfamiliar with these concepts are referred to [26] and [44].

\section{A. The Concept of Convolution on Groups}

Recall that the input-output relationship of a stable linear system can be represented by the following integral equation:

$$
y(t)=\int_{-\infty}^{\infty} K(t, \tau) x(\tau) d \tau
$$

where $y$ and $x$ are finite energy signals, i.e., elements of $L^{2}(\mathbb{R}, d t)$. If we assume that the linear system is time invariant, the kernel $K$ of the system has to satisfy the following invariance relationship:

$$
K(t, \tau)=K(t+T, \tau+T), \quad \text { for all } \infty<t, \tau, T<\infty .
$$

As a result, kernel $K$ can be simplified as

$$
k(t-\tau) \equiv K(t, \tau)=K(t-\tau, 0)
$$

and the input-output relationship of a linear time-invariant system can be represented as a convolution integral

$$
(x * k)(t)=\int_{-\infty}^{\infty} x(\tau) k(t-\tau) d \tau .
$$

The fundamental property of an ordinary convolution integral is its invariance under time shifts. To generalize the ordinary convolution to functions on groups, an appropriate integration measure invariant under group translations must be defined, i.e.,

$$
\int_{G} f(g) d \mu(g)=\int_{G} f(g \circ h) d \mu(g)
$$

for all $h$ in $G$ and $f$ integrable. For locally compact groups, such an integration measure exists and is called the (left) Haar measure. For any fixed element $h$ of $G$, the left Haar measure satisfies $d \mu(h \circ g)=d \mu(g)$, while the right Haar measure satisfies $d \mu_{R}(g \circ h)=d \mu_{R}(g)$. In general, left and right Haar measures are not the same for an arbitrary group. However, one has $d \mu_{R}(g)=\Delta^{-1}(g) d \mu(g)$, where $\Delta(g)$ is the modular function satisfying $\Delta(e)=1, \Delta(g)>0, \Delta(g \circ h)=$ $\Delta(g) \Delta(h)$. Those groups for which the modular function is 1 are called unimodular. For example, the Euclidean motion group and the Heisenberg group are unimodular, but affine and scale Euclidean groups are nonunimodular.

Analytical developments associated with the right Haar measure can be easily deduced from the results associated with the left Haar measure. Therefore, for the rest of this paper, we shall use the left Haar measure, unless specified otherwise. To simplify notation, we shall denote left Haar measure by $d g \equiv d \mu(g)$.

Let $L^{2}(G, d g)$ denote the Hilbert space of all complex-valued, square-integrable functions on the group $G$, and let $x$ and $y$ be two square integrable signals, representing the input and output of a linear system. Then, the input-output relationship of the system can be represented by the following integral:

$$
y(g)=\int_{G} K(g, h) x(h) d h
$$

where $K$ is a kernel defined on $G \times G$ and $d h$ is the left Haar measure of the group. Now, let us assume that the system $S$ has the following group-invariant dynamics:

$$
S\{x(g)\}=y(g) \Rightarrow S\{x(h \circ g)\}=y(h \circ g)
$$

for all $g, h$ of the group $G$. It is straightforward to show that such a group-invariant dynamic is satisfied if and only if the kernel $K$ satisfies

$$
K(g, h)=K(p \circ g, p \circ h)
$$

for any $p$ of the group $G$. Thus, the kernel $K$ can be reduced to a function of $G$

$$
k\left(h^{-1} \circ g\right) \equiv K\left(h^{-1} \circ g, e\right)=K(g, h)
$$

and, the input-output relationship in (3.3a) reduces to a convolution integral over the group $G$

$$
(x * k)(g) \equiv y(g)=\int_{G} x(h) k\left(h^{-1} \circ g\right) d h .
$$


For a square integrable function on an arbitrary Lie group, $f\left(h^{-1} \circ g\right)$ is called a translation in the same sense that $f(t-\tau)$ is a translation of a function defined on the real line. In particular, $[L(h) f](g)=f\left(h^{-1} \circ g\right)$ is called a left regular representation while $[R(h) f](g)=f(g \circ h)$ is called a right regular representation. For the definition of group representations, see Appendix I. These representations indicate the order in which the elements appear in the group composition law. In general, the left and right translations are not equal. As a result, the convolution operation is not commutative for arbitrary groups. Obviously, for commutative groups convolution operation is commutative.

Note that convolution integral can also be expressed in terms of the left regular representation of the group

$$
(x * k)(g)=\langle L(g) k, \quad \bar{x}\rangle
$$

where $\bar{x}$ is the complex conjugate of the function $x$.

\section{B. Fourier Analysis on Groups}

The Fourier analysis on groups is closely associated with the theory of group representations. This theory, particularly for Lie groups, has many physical applications. From the perspective of group representation theory, Fourier analysis deals with the characterization of unitary representations as a direct sum (integral) of the irreducible unitary group representations. This characterization is then utilized to define the Fourier transformation, which has the property of mapping the convolution integral to multiplication in the transform domain. However, for an arbitrary group, such a characterization is far from unique. Nonetheless, considerably satisfactory results can be obtained if some restrictions are imposed on the group structure. It was shown that if the group $G$ is a separable, locally compact group of Type I, [45] unique characterizations of the unitary representations can be obtained in terms of the irreducible unitary representations of the group. These conditions on the group $G$ are satisfied by a large class of groups, including compact groups, separable locally compact commutative groups, all connected semi-simple Lie groups, unimodular Lie groups, all algebraic Lie groups, and the majority of well-behaved locally compact groups. [46], [47]. Fortunately, most of the noncommutative groups of interest in engineering applications, such as the Euclidean motion groups, the affine group, and the Heisenberg group, fall into this category and admit unique Fourier decomposition of functions. Here, we shall review the Fourier analysis on compact Lie groups and separable locally compact groups of Type I for both unimodular and nonunimodular case.

Let $U(g, \lambda)$ be the $\lambda$ th irreducible unitary representation of a separable locally compact group of Type I. Then, the operatorvalued Fourier transform on $G$ maps each $f$ in $L^{2}(G, d g)$ to the family $\{\hat{f}(\lambda)\}$ of bounded operators, where each $\hat{f}(\lambda)$ is defined by

$$
\mathcal{F}(f)(\lambda)=\hat{f}(\lambda)=\int_{G} f(g) U\left(g^{-1}, \lambda\right) d g
$$

or in component form

$$
\hat{f}_{i, j}(\lambda)=\int_{G} f(g) U_{i, j}\left(g^{-1}, \lambda\right) d g
$$

where $U_{i, j}(g, \lambda)$ are the $(i, j)$ th matrix elements of $U(g, \lambda)$. The collection of all $\lambda$ values is denoted by $\hat{G}$ and is called the dual of the group $G$. The collection of Fourier transforms $\{\hat{f}(\lambda)\}$ for all $\lambda \in \hat{G}$ is called the spectrum of the function $f$. The Fourier transform can be viewed as a one-to-one onto transformation from $L^{2}(G, d g)$ to $L^{2}(\hat{G}, d \nu)$ where $d \nu$ denotes the Plancherel measure in the space $\hat{G}$. The Fourier synthesis and the Plancherel formula differ somewhat depending on the group structure.

An important property of the operator-valued Fourier transform, reminiscent of the classical Fourier transform over the reals, is that the group convolution becomes operator multiplication on the Fourier side; more precisely

$$
\mathcal{F}\left(f_{1} * f_{2}\right)=\mathcal{F}\left(f_{2}\right) \mathcal{F}\left(f_{1}\right) .
$$

For locally compact commutative groups, all irreducible representations of the group are one dimensional. Hence, the Fourier spectrum is scalar valued and appears similar to the classical Fourier transform. The inversion formula, in this case, is given by [48].

$$
f(g)=\int_{\hat{G}} \hat{f}(\lambda) U(g, \lambda) d \lambda .
$$

The Plancherel formula is given by

$$
\int_{G}|f(g)|^{2} d g=\int_{\hat{G}}|\hat{f}(\lambda)|^{2} d \lambda .
$$

For compact groups (in particular, for finite groups), the matrix elements of the irreducible representations form a complete orthonormal basis for $L^{2}(G, d g)$. As a result, the following inversion formula holds [40]:

$$
f(g)=\sum_{\lambda \in \hat{G}} d(\lambda) \operatorname{trace}(\hat{f}(\lambda) U(g, \lambda))
$$

or in component form

$$
f(g)=\sum_{\lambda \in \hat{G}} \sum_{i, j=1}^{d(\lambda)} d(\lambda) \hat{f}_{i, j}(\lambda) U_{i, j}(g, \lambda)
$$

where $d(\lambda)$ denotes the dimension of the representation $U(g, \lambda)$. The Plancherel formula is given by

$$
\int_{G}|f(g)|^{2} d g=\sum_{\lambda \in \hat{G}} d(\lambda)\|\hat{f}(\lambda)\|^{2}
$$

For the case of separable locally compact groups of Type I, the Fourier inversion and the Plancherel formulas are given by [49]

$$
f(g)=\int_{\hat{G}} \operatorname{trace}\left(\left[\hat{f}(\lambda) C_{\lambda}^{-2}\right] U^{\dagger}(g, \lambda)\right) d \nu(\lambda)
$$

and

$$
\begin{aligned}
& \int_{G}|f(g)|^{2} d g \\
& \quad=\int_{\hat{G}} \operatorname{trace}\left(\left[\hat{f}(\lambda) C_{\lambda}^{-1}\right]\left[\hat{f}(\lambda) C_{\lambda}^{-1}\right]^{\dagger}\right) d \nu(\lambda)
\end{aligned}
$$

where $\hat{f}^{\dagger}(\lambda)$ denotes the adjoint of $\hat{f}(\lambda)$ and $d \nu(\lambda)$ is the Plancherel measure and $\left\{C_{\lambda}\right\}$ is a family of Hermitian posi- 
tive-definite operators with densely defined inverses such that the followings hold:

- $\left\{\hat{f}(\lambda) C_{\lambda}\right\}$ is trace class for each $\lambda \in \hat{G}$ and

- $U(g, \lambda) C_{\lambda} U^{\dagger}(g, \lambda)=\Delta^{1 / 2}(g) C_{\lambda}$

where $\Delta(g)$ is the modular function of the group $G$.

For a given group, both the family of operators $\left\{C_{\lambda}\right\}$ and the Plancherel measure can be determined uniquely. When the group is unimodular, $C_{\lambda}=I_{\lambda}$. Thus, the inversion and the Plancherel formulae become

$$
f(g)=\int_{\hat{G}} \operatorname{trace}\left(\hat{f}(\lambda) U^{\dagger}(g, \lambda)\right) d \nu(\lambda)
$$

and

$$
\int_{G}|f(g)|^{2} d g=\int_{\hat{G}} \operatorname{trace}\left(\hat{f}(\lambda) \hat{f}^{\dagger}(\lambda)\right) d \nu(\lambda)
$$

where $d \nu(\lambda)$ is the Plancherel measure.

As an example, we present the Fourier transform over the affine group or the $a x+b$ group. The affine group of the real line has two nonequivalent infinite-dimensional unitary irreducible representations. We denote them by $U_{ \pm}$. Let $U_{ \pm}$act on the Hilbert space $H_{ \pm}$that consists of functions $\psi_{ \pm}$whose Fourier transform are supported on the right and left half half-line, respectively

$$
U_{ \pm}(a, b) f(x)=\frac{1}{\sqrt{a}} \psi_{ \pm}\left(\frac{x-b}{a}\right) .
$$

Then, the Fourier transform of a function defined on the affine group, i.e., $f \in L^{2}\left(\mathbb{R} \times \mathbb{R}^{+} a^{-2} d a d b\right)$ is given by the following operator valued functions:

$$
\mathcal{F}_{U_{ \pm}}(f)=\int_{-\infty}^{\infty} \int_{0}^{\infty} a^{-2} d a d b f(a, b) U_{ \pm}(a, b)
$$

where $a^{-2} d a d b$ is the left Haar measure of the affine group. The Fourier inversion formula is given by

$$
f(a, b)=\sum_{ \pm} \operatorname{trace}\left(U_{ \pm}^{\dagger}(a, b) \mathcal{F}_{U_{ \pm}}(f) \delta\right) .
$$

Note that the linear operator $\delta$ is given by

$$
\delta \xi(t)=\tilde{\mathcal{F}}^{-1}[|\omega| \tilde{\mathcal{F}}\{\xi\}(\omega)](t)
$$

where $\xi \in L^{2}(\mathbb{R}, d t)$ and $\tilde{\mathcal{F}}$ is the Fourier transform of the additive group [20]. The extra operator $\delta$ in the affine group Fourier inversion formula is due to the nonunimodular nature of the affine group.

In the following section, Fourier decomposition discussed in this section will be utilized to develop spectral decomposition theorems for a class of nonstationary stochastic processes.

\section{Group Stationary Processes}

One of the key components of our development is the generalized second-order stationary processes indexed by topological groups [50]-[52]. These processes are nonstationary in the classical sense but exhibit invariance under the right or left regular transformations of the group. Obviously, ordinary stationary processes are group stationary in which the underlying group is the additive group $(\mathbb{R},+)$. The author demonstrated in her earlier work that the special cases of group stationary processes for the multiplicative and affine group form suitable mathematical frameworks for modeling and analysis of self-similar and multiscale processes. Formally, the group stationary processes are defined as follows.

Let $G$ denote a group $g, h$ be its elements. Then, the process $X(g), g \in G$ is group stationary if

$$
X(g) \equiv X(h \circ g), \quad g, h \in G
$$

or

$$
X(g) \equiv X(g \circ h), \quad g, h \in G
$$

where $\equiv$ denotes equality in terms of finite joint probability distributions. Depending on whether a random process satisfies (4.1a), (4.1b) or both, it is called left, right, or two-way group stationary. Note that for commutative groups, the process is always two-way group stationary.

Second-order group stationarity is a weaker condition in which, only the second order statistics of the random process is required to be invariant under the right or left regular transformations of the group. Loosely speaking, second-order group stationary processes obey the following structure:

$$
E[X(g) \overline{X(h)}]=R\left(g \circ h^{-1}\right), \quad g, h \in G
$$

where $R$ is a positive-definite function defined on the group

The central fact in the analysis of group stationary processes is the existence of spectral decomposition, which is facilitated by the Fourier theory on groups. For compact groups, left group stationary processes admit the following spectral decomposition:

$$
X(g)=\sum_{\lambda \in \hat{G}} \operatorname{trace}(U(g, \lambda) Z(\lambda))
$$

and

$$
R(g)=\sum_{\lambda \in \hat{G}} \operatorname{trace}(U(g, \lambda) F(\lambda))
$$

with

$$
\sum_{\lambda \in \hat{G}} \operatorname{trace}(F(\lambda))<\infty
$$

where $R(\cdot)$ is the autocorrelation function of the process, $U(g, \lambda)$ is the $\lambda$ th irreducible unitary representation of the group $G$ with dimension $d(\lambda), Z(\lambda)$ is a random matrix of dimension $d(\lambda)$, and $F(\lambda)$ is a bounded Hermitian positive-definite operator over $\hat{G}$.

For separable locally compact groups of Type I, the summation in (4.3a) is replaced by an integral and the left group stationary processes admit the following spectral decomposition:

$$
X(g)=\int_{\hat{G}} \operatorname{trace}(U(g, \lambda) Z(d \lambda))
$$

and

$$
R(g)=\int_{\hat{G}} \operatorname{trace}(U(g, \lambda) F(d \lambda))
$$


where $R$ and $U(g, \lambda)$ are as described above, $Z(d \lambda)$ is a random linear operator over $\hat{G}$, and $F(d \lambda)$ is a operator measure over $\hat{G}$ satisfying

$$
\int_{\hat{G}} \operatorname{trace}(F(d \lambda))<\infty .
$$

The random linear operator $Z(\cdot)$ can be thought of as a matrix in which each matrix element is a random variable. In the case of commutative groups, this matrix is a diagonal one and the matrix elements are statistically independent. In the case of noncommutative groups, it is typically a block-diagonal matrix in which the matrix elements from one block to another are independent, but not necessarily independent within the same block. Unless the process is both right and left group stationary, the matrix entries of the random linear operator $Z(\cdot)$ is column-wise correlated and row-wise uncorrelated with correlation coefficients equal to the corresponding matrix entries of the operator $F(\cdot)$. For a rigorous treatment of the topic, we refer the reader to [50], [51].

Now, let us assume that the autocorrelation function $R$ is a square integrable function defined on the group $G$, i.e.,

$$
\int_{G}|R(g)|^{2} d g<\infty \text {. }
$$

We define

$$
S(\lambda) \equiv \mathcal{F}(R)(\lambda)=\int_{G} d g R(g) U(g, \lambda) .
$$

We shall refer to $S$ as the spectral density function of a group stationary process. It is straightforward to show that $S$ is a bounded nonnegative-definite operator or matrix-valued function defined on the dual space $\hat{G}$. This is a natural generalization of the spectral density function defined for ordinary stationary processes due to its generalized version of symmetry and positivity properties. Obviously, the operator-valued spectral density function is closely related to the operators defined in (4.3b) and (4.4b). For compact groups, they are equal and for noncompact groups, loosely speaking, spectral density function is associated with the derivative of the operator measure defined in (4.3b) and (4.4b). Hence, the spectral density function represents the correlation structure of the underlying elementary almost white random operators $Z(\cdot)$. We shall utilize the concept of spectral density function extensively in our subsequent development.

For practical implementation and processing of time series with group-invariant structure, one has to define a notion of ergodicity. See Wiener [83] for the generalized notion of ergodicity over groups.

\section{Examples:}

For commutative groups, irreducible unitary representations of the group are one dimensional. As a result, the Fourier transform diagonalizes the covariance function, but for noncommutative groups, the covariance function can only be block diagonalized.

\section{A. Shift Stationary Processes}

The simplest example of the group stationary processes is the ordinary stationary processes defined on the real line with the addition operation, i.e., the additive group $(\mathbb{R},+)$. For the rest of our discussion, we shall refer to these processes as shift stationary processes

$$
E\left[X\left(t_{1}\right) \overline{X\left(t_{2}\right)}\right]=R\left(t_{1}-t_{2}\right), \quad-\infty<t_{1}, t_{2}<\infty .
$$

One-dimensional irreducible representations of the additive group $(\mathbb{R},+)$ are given by the complex exponential functions $e^{j \omega t},-\infty<t_{1}, t_{2}<\infty$. Hence, the whitening transform, or the spectral decomposition of the shift stationary processes is given by the ordinary Fourier transform. As a result of the well-developed analytical framework based on Fourier theory coupled with the existence of the fast Fourier algorithms, shift stationary signal modeling and analysis has been indispensable in engineering applications.

\section{B. Scale Stationary Processes [19]}

Another important class of group stationary processes is defined by the multiplicative group, on the positive real line, i.e., $\left(\mathbb{R}^{+}, \times\right)$. These processes exhibit invariance with respect to translation in scale, and are referred to as scale stationary processes [19]. Their second-order statistic obeys the following structure:

$$
E\left[X\left(t_{1}\right) \overline{X\left(t_{2}\right)}\right]=R\left(t_{1} / t_{2}\right), \quad 0<t_{1}, t_{2}<\infty .
$$

These processes and their mathematical framework are shown to be optimal for modeling and analysis of statistically self-similar phenomena [19]. Formally, self-similar processes are defined as follows:

$$
X(t) \equiv a^{-H} X(a t), \quad \text { for all } a>0
$$

where $\equiv$ denotes equality in probability distribution and $H$ is a constant parameter characterizing the degree of self-similarity. Scale stationary processes are self-similar with parameter 0 . Nevertheless, there is a one-to-one correspondence between a self-similar process with parameter $H$ and a scale stationary process defined through the following relationship:

$$
\tilde{X}(t)=t^{H} X(t), \quad t>0
$$

in which $\tilde{X}$ is self-similar with parameter $H$ if and only if $X$ is scale stationary. Fig. 1 shows the sample path of a scale stationary autoregressive moving average process. Smoothness of the sample paths over large time scales indicates the ability of scale stationary models in capturing long-term correlations.

One-dimensional irreducible representations of the multiplicative group are given by $e^{j \omega \log t}, t>0$. As a result, the Mellin transform whitens the scale stationary processes. Detailed analysis of the self-similar processes based on the concept of scale stationarity can be found in the present author's previous work [19], [21], [66], [67].

\section{Affine Stationary Processes [19], [21]-[23]}

These are second-order processes defined on the $a x+b$ or affine group, i.e., $X(a, b), a>0,-\infty<b<\infty$. Due to the noncommutativity of the affine group, stochastic processes induced by the left and right regular representation of the group are different, nevertheless related. Here, we shall only discuss the left affine stationary processes. 


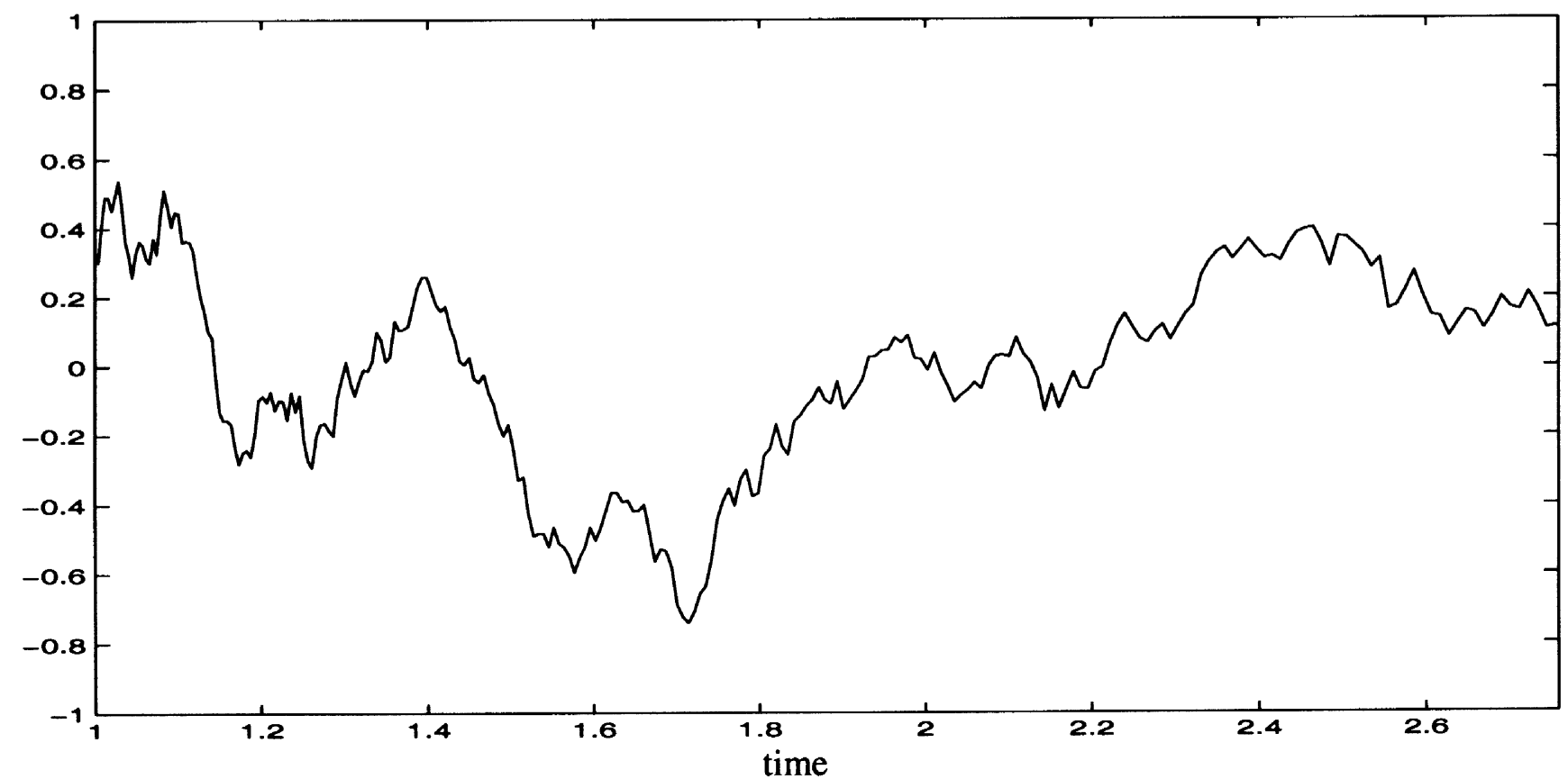

Fig. 1. Sample paths of first-order scale stationary autoregressive (SSAR) process with unit variance and parameter $\alpha=0.6$.

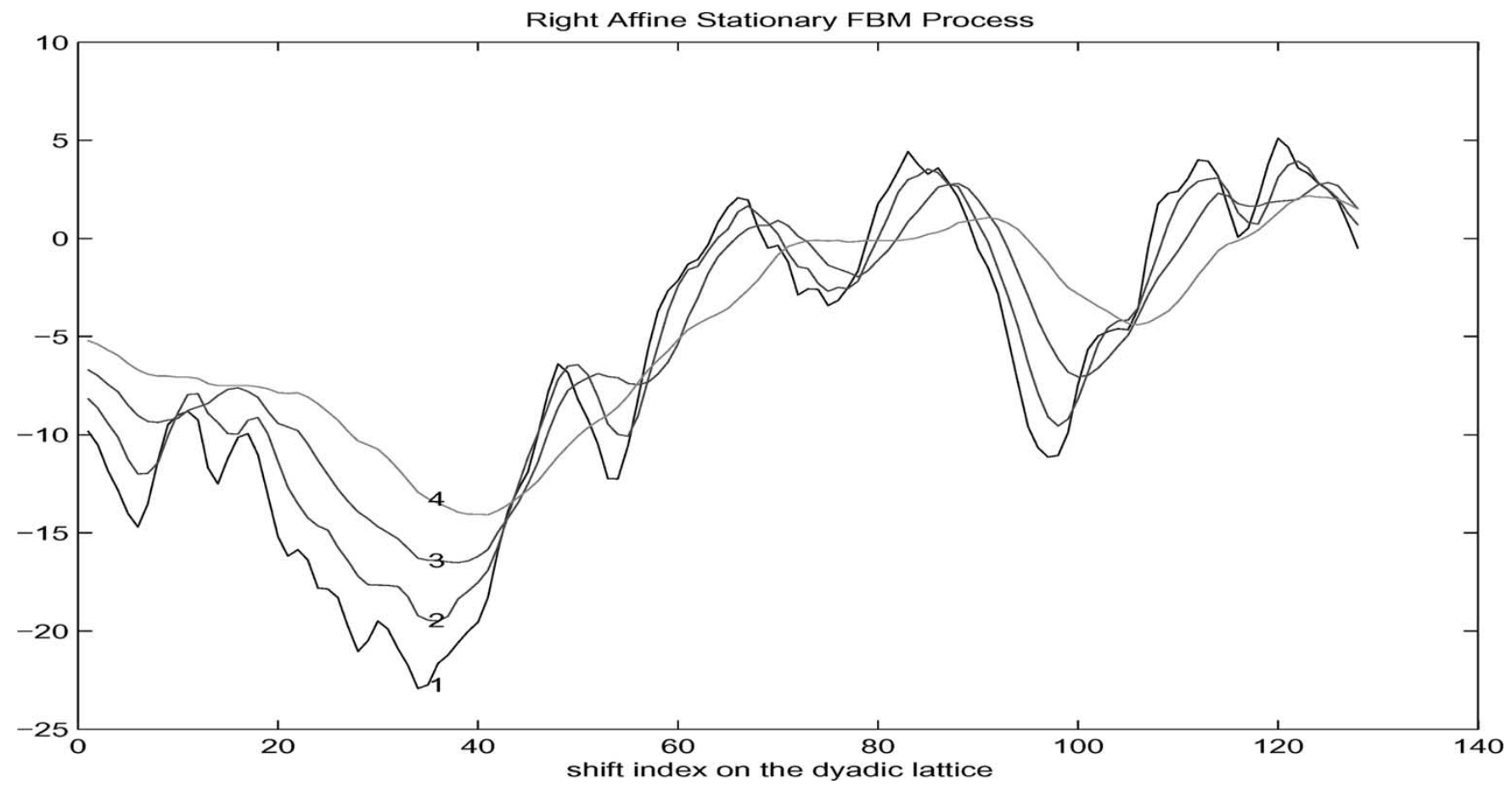

Fig. 2. Sample paths of an affine stationary process derived from fractional Brownian motion (fBM). Each line represents the sample paths at a different scale, level 1 being the lowest scale.

Affine stationary processes are proposed for statistical modeling of multiscale signals, such as random signals in the wavelet domain or signals obtained at different resolutions [20]. The index $a$ is interpreted as the scale, and the index $b$ is interpreted as the shift index. The autocorrelation function of a left affine stationary process is given as

$$
E\left[X\left(a_{1}, b_{1}\right) \overline{X\left(a_{2}, b_{2}\right)}\right]=R\left(\frac{a_{1}}{a_{2}}, \frac{1}{a_{2}}\left(b_{1}-b_{2}\right)\right) .
$$

Thus, a left affine stationary process is shift stationary within the same scale and scale stationary across the scales for fixed shift index. Sample paths of a left affine stationary process shown in Fig. 2 clearly indicate the strong correlation across the scales. It was also shown by the author that the spectral decomposition of affine stationary processes is associated with the wavelet transforms. For a detailed discussion on affine stationary processes and their spectral decomposition, see [19].

Apart from these examples, detailed study on stochastic processes invariant with respect to the two- and three-dimensional rotational group action can be found in [81] and [82] and references therein. 
Note that both the estimator/correlator output in radar/sonar imaging described in Section II-A and the tomographic imaging process described in Section II-B, can be viewed as convolution operations over homogenous spaces in the sense that the left regular representation in (3.5) is replaced by the quasi-left regular representation and the space $L^{2}(G)$ is replaced by the space of square summable functions on the homogeneous space $X$, i.e.,

$$
y(g)=\left\langle L_{q}(g) f \quad \bar{x}\right\rangle
$$

where $f$ and $x$ are elements of $L^{2}(X)$ and $L_{q}(g) f(t)=$ $f\left(g^{-1} t\right)$. Standard examples of the homogenous spaces are the sphere $S^{n}=S O(N+1) / S O(N)$ and the Euclidean spaces $\mathbb{R}^{N}=S E(N) / S O(N)$. In engineering practice, measurements are typically indexed by homogenous spaces. Therefore, it is useful to investigate the class of stochastic processes indexed by the homogeneous space $X$ that leads to group stationary processes after the "detection/imaging" process described by (4.10). To do so, we shall first define stochastic processes that are stationary on homogeneous spaces.

Let $X$ be a homogenous space on which the topological group $G$ is acting transitively, and let $\{x(t), t \in X\}$ be a stochastic process with constant mean and finite variance satisfying the following condition:

$$
E\left[x\left(g t_{1}\right) x\left(g t_{2}\right)\right]=E\left[x\left(t_{1}\right) x\left(t_{2}\right)\right]
$$

for all $t_{1}, t_{2}$ in $X$ and $g$ in $G$. Then, we shall call $\{x(t), t \in X\}$ a group stationary process on $X$. For spectral decomposition of such processes, see [50]. Loosely speaking, the following theorem states how group stationary processes are induced from stationary processes on homogeneous spaces via convolution operation.

Before we introduce the theorem, let us define the relatively invariant measure for homogeneous spaces. The homogenous space $X$ is said to possess a relatively invariant measure $\mu(d t)$ if there is a character $\rho_{t}(g)$ on $G$ such that

$$
\mu\left(d g^{-1} t\right)=\rho_{\mu}(g) \mu(d t)
$$

[75] i.e.,

$$
\rho_{t}(g) \int_{X} f\left(g^{-1} t\right) d t=\int_{X} f(t) d t, \quad \text { for } g \in G .
$$

Note that a relatively invariant measure on $X$ exists if and only if the modular function of $H$, where $X=G / H$, can be extended as a character of $G$ [75]. For example, it is sufficient for $H$ to be unimodular.

Theorem 1: Let $X$ be a homogenous space on which the topological group $G$ is acting transitively, and let $\{x(t), t \in X\}$ be a stochastic process on $X$. Assume that the homogeneous space $X$ has a relatively invariant measure $\mu(d t)$. Let

$$
y(g)=\int_{X} L_{q}(g) f(t) x(t) \mu(d t)
$$

where $f$ is an element of $f \in L^{2}(X, \mu(d t)) \cap L(X, \mu(d t))$ and $L_{q}(g)$ is the quasi-left regular representation of the group

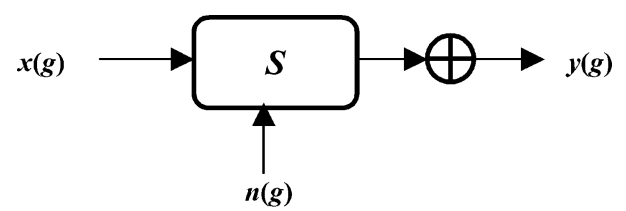

Fig. 3. Observation model.

$G$ on $L^{2}(X, \mu(d t))$. Then, $y(g), g \in G$ is group stationary up to an amplitude factor if and only if $\{x(t), t \in X\}$ is group stationary on the homogenous space $X$. In this case, the secondorder statistics of the process $y(g), g \in G$ has the following structure:

$$
E\left[y\left(g_{1}\right) y\left(g_{2}\right)\right]=\rho_{\mu}^{2}(g) E\left[y\left(g \circ g_{1}\right) y\left(g \circ g_{2}\right)\right]
$$

where $\rho_{\mu}(g)$ is the character on $G$ such that $\mu\left(d g^{-1} t\right)=$ $\rho_{\mu}(g) \mu(d t)$.

Proof: See Appendix II.

A trivial process that is group stationary on homogenous spaces is the "white noise" process that can be rigorously defined via orthogonal random set functions. Let $\{Z(B), B \in \boldsymbol{B}\}$ be an orthogonal random set function satisfying

$$
E\left[Z\left(B_{1}\right) Z\left(B_{2}\right)\right]=\mu\left(B_{1} \cap B_{2}\right)
$$

where $\boldsymbol{B}$ is a $\sigma$-field of the homogenous space $X$ and $\mu$ is the measure on $(X, B)$. By definition, the orthogonal process is stationary on $X$ since the measure $\mu$ is invariant under left translations. Thus, the following process is left group stationary up to an amplitude factor:

$$
y(g)=\int_{X} L_{q}(g) f(t) d Z(t)
$$

where $f$ is an element of $f \in L^{2}(X, d \mu) \cap L(X, d \mu)$, and $L_{q}(g)$ is the quasi-left regular representation of the group $G$ in $L^{2}(X, d \mu)$.

In engineering applications, often the homogenous space of interest is either $\mathbb{R}^{N}$ or $\mathbb{C}^{N}$, in which case, the process with orthogonal increments is the Brownian motion process. Thus, the theorem states that the filtered "white noise" process has a group stationary structure.

\section{WIENER FILTERING OVER GROUPS}

In this section, we shall introduce a novel stochastic deconvolution method over groups based on the Fourier theory of the topological groups. We shall pose the deconvolution problem within the framework of minimum mean-square error (MMSE) prediction, and develop a Wiener filtering method to estimate unknown signals from noisy measurements. While our results will be stated for the locally compact groups of Type I, special cases of finite, compact, and commutative groups can be easily deduced from the main result.

Let $S$ be a left group-invariant system defined on a locally compact group $G$ of Type I, and let $y$ be the noisy output of the system for an input $x$ as depicted in Fig. 3 . 
Thus, the observation model is given by the following convolution integral:

$$
y(g)=\int_{G} x(h) f\left(h^{-1} \circ g\right) d h+n(g)
$$

where $f: G \rightarrow \mathbb{C}$ is the complex-valued, square-summable impulse response function of the group-invariant system $S$, and $x$ and $n$ are random processes of the unknown signal and additive noise indexed by the group $G$, taking values in the field of complex numbers $\mathbb{C}$. Without loss of generality, we assume that $E[x(g)]=E[n(g)]=0$. Then, the classical linear Wiener problem of recovering $x$ from noisy measurements $y$ can be posed as follows: Find the linear filter $W: G \times G \rightarrow \mathbb{C}$ such that the least squares error variance

$$
J\left(\varepsilon_{w}\right)=\int_{G} E\left[\left|\varepsilon_{w}(g)\right|^{2}\right] d g
$$

is minimized, where

$$
\varepsilon_{w}(g)=\int_{G} W(g, h) y(h) d h-x(g),
$$

and $d h$ is the left Haar measure on the group $G$. Note that it is implicit by the (5.2a) that the filter $W$ must be doubly square summable. Then, the solution to the above linear least squares problem is provided by the following Wiener-Hopf type equation:

$$
\int_{G} W(g, s) R_{y y}(s, h) d s=R_{x y}(g, h)
$$

where

$$
R_{y y}(s, h)=E[y(s) \overline{y(h)}]
$$

and

$$
R_{x y}(g, h)=E[x(g) \overline{y(h)}]
$$

Alternatively

$$
\left(\tilde{f} * R_{x}\right)(g)=\int_{G} R_{y}(p) \tilde{W}\left(p^{-1} \circ g\right) d p
$$

where

$$
\begin{aligned}
& R_{x}(g)=R_{x x}(g, e)=E[x(g) \overline{x(e)}] \\
& R_{y}(g)=R_{y y}(g, e)=E[y(g) \overline{y(e)}]
\end{aligned}
$$

and

$$
\tilde{W}(g)=W(g, e) \quad \text { and } \quad \tilde{f}(g)=\overline{f\left(g^{-1}\right)} .
$$

The following theorem states an explicit solution for the Wiener-Hopf equation, which in turn leads to the linear least squares recovery of the signal $x$.

Theorem 2: Let $G$ be a separable locally compact group of Type I, and $x(g)$ and $n(g), g$ of $G$, be two zero mean left group stationary processes, referred to as signal and noise, respectively. Assume that the measurements obey the following convolution integral and noise model:

$$
y(g)=\int_{G} x(h) f\left(h^{-1} \circ g\right) d h+n(g)
$$

and

$$
E[x(g) \overline{n(g)}]=0
$$

where $f \in L^{2}(G, d g)$. Then, the optimum linear least squares deconvolution filter $W_{\text {opt }}$, minimizing (5.2a), is left group invariant and the estimate of the signal is given as a convolution integral

$$
\hat{x}(g)=\int_{G} y(h) W_{\text {opt }}\left(h^{-1} \circ g\right) d h .
$$

The Fourier transform of the optimal filter $W_{\text {opt }}$ is given by

$$
\begin{array}{r}
\hat{W}_{\text {opt }}(\lambda)=S_{x}(\lambda) \hat{f}^{\dagger}(\lambda)\left[\hat{f}(\lambda) S_{x}(\lambda) \hat{f}^{\dagger}(\lambda)+S_{n}(\lambda)\right]^{-1}, \\
\lambda \in \hat{G} . \quad(5.5 \mathrm{~d})
\end{array}
$$

Here, $\hat{f}$ is the Fourier transform of the convolution filter $f$, and $\hat{f}^{\dagger}$ denotes the adjoint of the operator $\hat{f}$. $S_{x}$ and $S_{n}$ are operator-valued spectral density functions of the signal and noise, respectively. The spectral density function of the least square error between the signal and its filtered estimate is given as

$$
S_{\varepsilon}(\lambda)=\left(I-\hat{W}_{\text {opt }}(\lambda) \hat{f}(\lambda)\right) S_{x}(\lambda)
$$

where $I$ denotes the identity operator.

Proof: See Appendix II.

\section{Remarks:}

Note that $\hat{f}(\lambda) S_{x}(\lambda) \hat{f}^{\dagger}(\lambda)+S_{n}(\lambda)$ is a nonnegative definite operator. Thus, its inverse exists, but may be unbounded. In that case, $\left[\hat{f}(\lambda) S_{x}(\lambda) \hat{f}^{\dagger}(\lambda)+S_{n}(\lambda)\right]^{-1}$ can be interpreted as the pseudo-inverse. When the underlying group is the additive group of reals, $(\mathbb{R},+)$, the Wiener filter given in $(5.5 \mathrm{~d})$ becomes the classical Wiener filter given by

$$
\hat{W}_{\mathrm{opt}}(\omega)=\frac{S_{x}(\omega) \overline{\hat{f}}(\omega)}{S_{x}(\omega)|\hat{f}(\omega)|^{2}+S_{n}(\omega)}
$$

where

$$
\hat{f}(\omega)=\int_{-\infty}^{\infty} f(t) e^{-j \omega t} d t .
$$

Similar to the classical Wiener filtering, the results stated in Theorem 2 can be extended to the case where the signal and the noise are correlated. Under the assumption that the noise and the signal are jointly left group stationary, i.e.,

$$
E\left[x\left(g_{1}\right) \overline{n\left(g_{2}\right)}\right]=E\left[x\left(h \circ g_{1}\right) \overline{n\left(h \circ g_{2}\right)}\right]
$$

for any $g_{1}, g_{2}$, and $h$ element of the group $G$, it is straightforward to show that the Wiener-Hopf equation in (5.3) becomes a group convolution integral which can be solved in a manner similar to the case described in Theorem 2 above.

In the absence of a blurring filter $f$, the optimal filter which separates the signal from the noise can be defined in terms of an operator-valued signal-to-noise ratio function

$$
\hat{W}_{\mathrm{opt}}(\lambda)=S_{\mathrm{SNR}}(\lambda)\left[S_{\mathrm{SNR}}(\lambda)+I\right]^{-1}
$$

where

$$
S_{\mathrm{SNR}}(\lambda)=S_{x}(\lambda) S_{n}(\lambda)^{-1}
$$


This formulation of the optimal filter does not require the signal and noise spectral density functions and may be preferable over the formulation in (5.5d) if the signal-to-noise spectral density function can be developed from the system and physical modeling of the problem.

Note that the proposed Wiener filter provides a regularized solution to the inversion problem. To see this, let us assume that there is no noise in measurements, then the Wiener filter becomes $\hat{W}_{\text {opt }}=\hat{f}^{-1}(\lambda)$. However, the Fourier transform of the blurring filter $f$ may not be invertable if it has singularities. On the other hand, $\hat{f}(\lambda) S_{x}(\lambda) \hat{f}^{\dagger}(\lambda)+S_{n}(\lambda)$ is always a nonnegative-definite operator. With appropriate choice of prior and noise model, one can assure that it is a positive-definite matrix with eigenvalues away from zero. For example, when the measurement noise is "white" with variance $\sigma^{2}$, i.e., $S_{n}(\lambda)=$ $\sigma^{2} I(\lambda), \hat{f}(\lambda) S_{x}(\lambda) \hat{f}^{\dagger}(\lambda)+\sigma^{2} I(\lambda)$ has eigenvalues away from zero which coincides with the Tikhonov regularization of order zero [54].

Another case is when there is no noise in measurements and no prior knowledge on the statistical distribution of the unknown signal is available. For simplicity, we can assume that the spectral density function of the unknown signal is simply white noise with unit variance. In this case, the Wiener filter is simply the minimum norm linear least squares filter given by

$$
\hat{W}_{\text {opt }}(\lambda)=\hat{f}^{\dagger}(\lambda)\left[\hat{f}(\lambda) \hat{f}^{\dagger}(\lambda)\right]^{-1} .
$$

However, this estimate is unstable in the sense that small deviations in measurements may lead to large fluctuations in the estimation due to the eigenvalues of $\hat{f}(\lambda) \hat{f}^{\dagger}(\lambda)$ that are close to 0 . The zero-order Tikhonov regularization of the form

$$
\hat{W}_{\mathrm{opt}}(\lambda)=\hat{f}^{\dagger}(\lambda)\left[\hat{f}(\lambda) \hat{f}^{\dagger}(\lambda)+\sigma^{2} I(\lambda)\right]^{-1}
$$

which amounts to the case where no a priori information on the spectral density function of the unknown signal is available, i.e., $S_{X}(\lambda)=I(\lambda)$ and the spectral density function of noise is $S_{n}(\lambda)=\sigma^{2} I(\lambda)$. Note that in [56], Chirikjian et al. provided the solution in (5.8) as a solution to the convolution equation over the Euclidean motion groups, which is a special case of the proposed Wiener filtering method.

The minimum mean square solution of the convolution integral stated in Theorem 2 can easily be extended to integral equations involving representations that are equivalent to the left regular representation. The convolution integral is a result of the left regular representation of the group $G$ acting on function in $L^{2}(G, d g)$, i.e.,

$$
\left(f_{1} * f_{2}\right)(g)=\left\langle L(g) f_{2}, \bar{f}_{1}\right\rangle, \quad f_{1}, f_{2} \in L^{2}(G, d g)
$$

where $\bar{f}_{1}$ denotes the complex conjugate of $f_{1}$. Let $L_{H}(g)$ be a representation equivalent to the left regular representation acting on vectors in a Hilbert space $H$. Then, by definition, there is a linear operator $A$, mapping $H$ into $L^{2}(G, d g)$, with an inverse linear operator $A^{-1}$, such that

$$
A L_{H}(g) A^{-1}=L(g) .
$$

The solution of the integral equation

$$
\begin{aligned}
& f_{3}(g)=\left\langle L_{H}(g) \tilde{f}_{2}, \tilde{\tilde{f}}_{1}\right\rangle, \\
& \quad \tilde{f}_{1}, \tilde{f}_{2} \in H \text { and } f_{3} \in L^{2}(G, d g)
\end{aligned}
$$

is equal to the solution of

$$
f_{3}(g)=\left\langle L(g) A f_{2}, A \bar{f}_{1}\right\rangle, \quad f_{1}, f_{2}, f_{3} \in L^{2}(G, d g)
$$

where $A f_{2}=\tilde{f}_{2}$ and $A \bar{f}_{1}=\overline{\tilde{f}}_{1}$. Using the Hilbert space theory of stochastic processes, the argument described above can be extended to include group stationary random processes. Such an extension requires definition of an equivalence relationship for group stationary processes via the equivalence relationship of group representations. In defining an equivalence relationship for group stationary processes, Parzen's theory of associating a unique vector from the reproducing kernel Hilbert space generated by the covariance function of the random process is the key in bridging the random and deterministic signals [55].

\section{EXAMPLE: ReCOVERY OF BLURRED AND NOISY SELF-SiMILAR PROCESSES}

In this section, we will demonstrate the application of the proposed Wiener filtering method to the recovery of the blurred and noisy self-similar processes. One of the fundamental properties of the self-similar processes is their strong long-term correlation structure. Such a problem is commonly encountered in practice where both the signal and noise exhibit strong long-term correlations. There is a variety of self-similar models that capture such long-term correlations parsimoniously, including fractional Brownian motion (fBM) and scale stationary processes [53], [19]. We have demonstrated in our earlier work that the notion of scale stationarity described in Section IV, Example 2 leads to a complete, engineering-oriented mathematical framework to model and analyze self-similar processes. These include spectral representation, autoregressive moving average (ARMA) modeling, Kalman filtering, and Wiener filtering as will be demonstrated here [19], [22], [66]-[68].

Let the observation model be

$$
y(t)=f * x(t)+n(t)
$$

where

$$
\begin{aligned}
E[x(t)] & =E[n(t)]=0, \\
E[x(t) n(t)] & =0, \quad \text { for all } t>0 \\
E\left[x\left(t_{1}\right) x\left(t_{2}\right)\right] & =R_{x}\left(t_{1} / t_{2}\right)
\end{aligned}
$$

and

$$
E\left[n\left(t_{1}\right) n\left(t_{2}\right)\right]=R_{n}\left(t_{1} / t_{2}\right), \quad \text { for all } t_{1}, t_{2}>0 . \quad \text { (6.1c) }
$$

Due to the commutativity of the underlying group, the spectral density function is scalar valued defined via Mellin transform. Thus, the MMSE filter is given by

$$
\hat{W}_{\text {opt }}(\omega)=\frac{\overline{F(\omega)} S_{x}(\omega)}{|F(\omega)|^{2} S_{x}(\omega)+S_{n}(\omega)}, \quad-\infty<\omega<\infty
$$


where

$$
\begin{aligned}
& S_{x}(\omega)=\int_{0}^{\infty} R_{x}(\lambda) \lambda^{-j \omega-1} d \lambda \\
& S_{n}(\omega)=\int_{0}^{\infty} R_{n}(\lambda) \lambda^{-j \omega-1} d \lambda
\end{aligned}
$$

and $F(\omega)$ is the transfer function of the blurring filter in Mellin domain.

\section{A. Example 1}

For the first simulation, we model the signal as a first-order scale stationary autoregressive (SSAR) model given by

$$
t \frac{d x}{d t}(t)+\alpha x(t)=\sigma_{x} t^{H} w(t), \quad t>0
$$

where $w(t), t>0$ is a scale stationary "white noise" process and $\alpha$ is the scale stationary AR model parameter, and $H$ is the self-similarity parameter [19]. It is straightforward to show that the spectral density function of the first-order SSAR process is given by

$$
S_{x}(\omega)=\frac{\sigma_{x}^{2}}{\omega^{2}+\alpha^{2}}, \quad-\infty<\omega<\infty .
$$

We model the observation as a first-order SSAR model with parameter $\alpha=0.6$ and $H=0$ embedded in additive white Gaussian noise with variance $\sigma_{n}^{2}$

$$
y(t)=x(t)+n(t) .
$$

Clearly, the standard $(\mathbb{R},+)$ Fourier-based Wiener filter is not applicable for self-similar signals due to their nonstationary nature in the classical sense. Given the underlying multiplicative structure, the optimal MMSE filter in the Mellin domain is given by

$$
W_{\mathrm{opt}}(\omega)=\frac{\sigma_{x}^{2}}{\sigma_{n}^{2}\left(\omega^{2}+\alpha^{2}\right)+\sigma_{x}^{2}}
$$

where $\sigma_{n}^{2}$ is the variance of the additive noise. This filter is implemented in the Mellin domain and the time-domain estimate of the signal is obtained by taking the inverse Mellin transform of the estimate. Implementation of the Mellin transform depends on the optimal sampling and the notion of bandlimitedness for signals indexed by the multiplicative group. Detailed discussion of these concepts and efficient implementation of the Mellin transform are described in the author's previous work, [21], [22], [66], and [68].

We performed 100 Monte Carlo simulations for each noise level and compute the average mean difference and variance between the original and recovered signal to quantify the goodness of the recovered signals. These results are tabulated in Table I. For visual illustration of the proposed self-similar signal recovery method, we present the sample paths of the original signal, noisy signal with $10-\mathrm{dB}$ SNR, and the recovered signal in Fig. 4(a)-(c), respectively

\section{B. Example 2}

In the second simulation, the signal is assumed to be the generating process of the $\mathrm{fBm}$ with a self-similarity parameter $H=0.6$ [19]. The observation is a blurred and noisy $\mathrm{fBm}$
TABLE I

Mean Error and Mean Square Error Out of 100 Monte Carlo Runs FOR THE RECOVERY OF THE FIRST-ORDER SSAR PROCESS EMBEDDED IN White GAUSSIAN NOISE AT VARIOUS LEVELS

\begin{tabular}{c|c|c|c|c}
\hline & $5 \mathrm{~dB}$ & $10 \mathrm{~dB}$ & $20 \mathrm{~dB}$ & $40 \mathrm{~dB}$ \\
\hline Mean error & 0.0749 & 0.0544 & 0.0271 & 0.0033 \\
\hline $\begin{array}{c}\text { Mean } \\
\text { square error }\end{array}$ & 0.0091 & 0.0048 & 0.0012 & 0.0 \\
\hline
\end{tabular}

TABLE II

Mean Error and Mean Square Error Out of 100 Monte Carlo Runs FOR THE RECOVERY OF THE BLURRED AND NOISY fBM PROCESS AT VARIOUS NOISE LEVELS

\begin{tabular}{c|c|c|c|c}
\hline & $5 \mathrm{~dB}$ & $10 \mathrm{~dB}$ & $20 \mathrm{~dB}$ & $40 \mathrm{~dB}$ \\
\hline Mean error & 0.0350 & 0.0251 & 0.0122 & 0.0027 \\
\hline $\begin{array}{c}\text { Mean } \\
\text { square error }\end{array}$ & 0.0023 & 0.0012 & 0.0004 & 0.0 \\
\hline
\end{tabular}

process in which the noise is a first-order SSAR process and the blurring is due to a "low-pass" scale-invariant filter defined as

$$
f(\omega)=\cos ^{2}\left(\frac{\omega}{2}\right), \quad-\infty<\omega<\infty
$$

Time-domain realization of this filter is shown in Fig. 5(b). The scale autocorrelation of the fBm process is given by [19], [16]

$$
R_{\mathrm{fBm}}(\lambda)=V_{H}\left[\cosh (H \ln \lambda)-(1 / 2)|2 \sinh (\ln \lambda / 2)|^{2 H}\right]
$$

where

$$
V_{H}=1 /(\sin \pi H \Gamma(2 H+1)), \quad 0<H<1 .
$$

The scale spectral density of the fBm process is given by [16]

$S_{\mathrm{fBm}}(\omega)=\frac{\Gamma(1-H+i \omega) \Gamma(1-H-i \omega)}{\Gamma(1 / 2+i \omega) \Gamma(1 / 2-i \omega)\left(H^{2}+\omega^{2}\right)}$,

$$
-\infty<\omega<\infty \text {. }
$$

The sample paths of the noise-free fBm process and blurred and noisy measurements with 10-dB SNR are shown in Fig. 5(a) and (c), respectively. Inserting (6.7), (6.9), and (6.4) into (6.2a), an analytic expression for the MMSE filter can be obtained. The transfer function of the optimal MMSE filter was numerically formed and denoising is performed in the Mellin domain. The sample paths of the original and the denoised signal are presented in Fig. 4(d). Error analysis is performed based on 100 Monte Carlo runs. The mean and the mean-square values of the error are given in Table II.

Note that for locally compact commutative and compact groups, the Fourier domain deconvolution method described in this study can be efficiently implemented by the fast Fourier algorithms [58], [59], [25], [27]-[29]. The study of noncommutative noncompact sampling theory, Fourier algorithm development is still a relatively new area for computational methods. It appears that for this case, the extension of the bandlimitedness idea has to be addressed differently depending on the structure of the noncommutative locally compact groups. In [58], it was shown that the sampling algorithm and the discretization of generalized continuous wavelet transforms are equivalent problems for the locally compact unimodular groups 


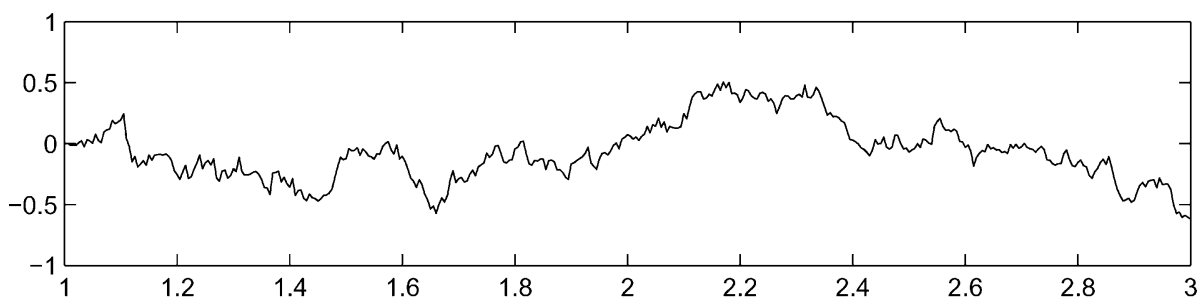

(a)

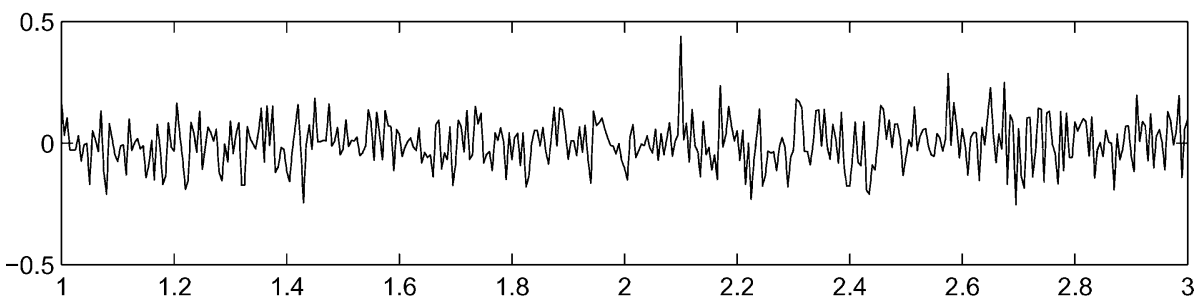

(b)

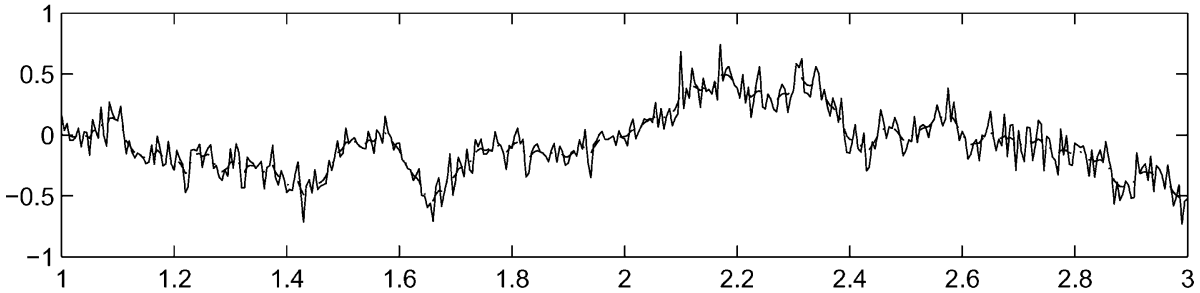

(c)

Fig. 4. (a) Sample paths of the first-order SSAR process with unit variance and $\alpha=0.6$. (b) First-order SSAR process in Fig. 3(a) embedded in white noise with $\mathrm{SNR}=10 \mathrm{~dB}$. (c) Sample path of the recovered process after Mellin based Wiener filtering. Solid line: recovered process; dashed line: original.

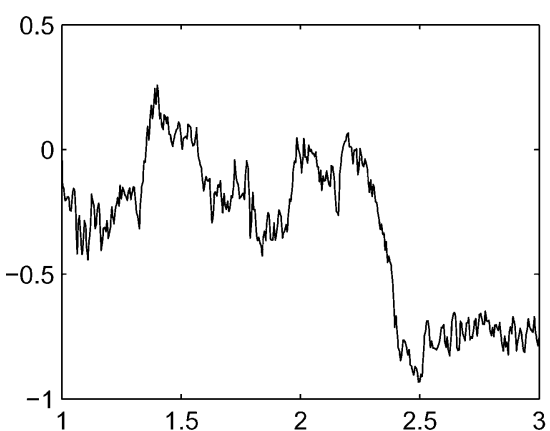

(a)

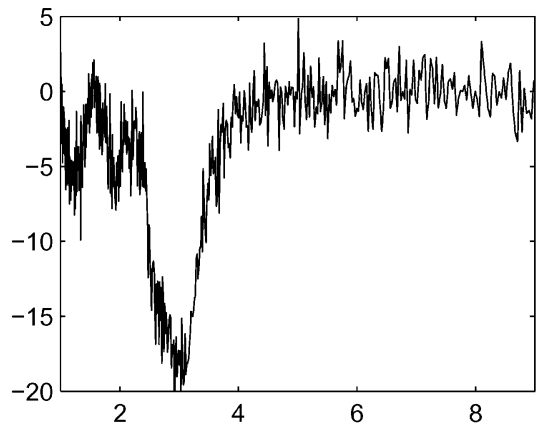

(c)

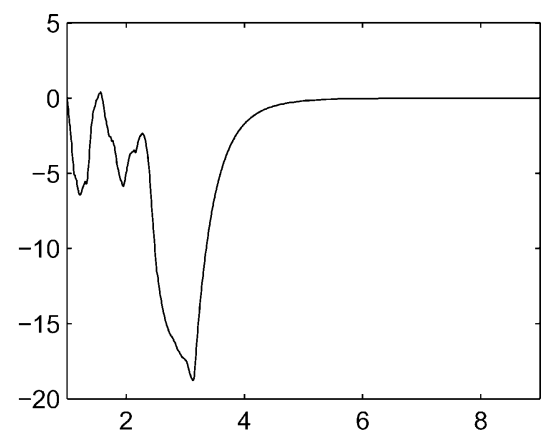

(b)

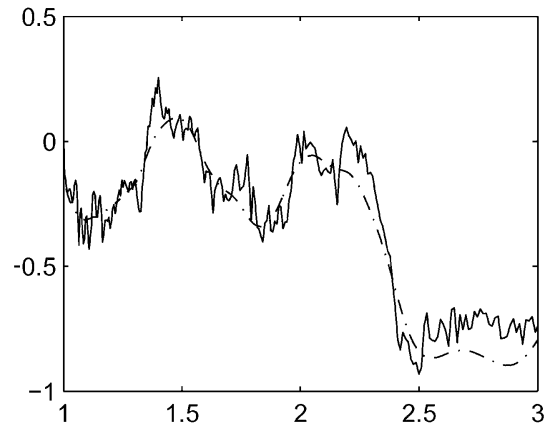

(d)

Fig. 5. (a) The generating process of the fBm process with $H=0.6$. (b) Time-domain representation of the blurring filter defined in (6.7). (c) Sample path of the blurred and noisy observations. (d) Sample paths of the original and recovered signal. Solid line: recovered process; dashed line: original. 
using the framework of Plancherel theory. See [58] for the implementation of sampling algorithms for Heisenberg group. In [72]-[74], a notion of bandlimitedness is defined and sampling theorems for functions on groups are developed.

Approximate methods of Fourier transform implementation can be addressed on a case-by-case basis based on the particular structure of the group. For example, in [60], the Fourier transform over the two-dimensional Euclidean motion group $S E(2)$ is approximated by the Fourier transform of the discrete motion group which is defined as the semi-direct product of the discrete rotation group and the additive group $R$. In [29], an approximate Shannon-type sampling theorem is derived for Euclidean motion groups.

\section{CONCLUSION}

In this paper, we have shown how group representation theory can be utilized to solve a class of inverse problems formulated as group convolutions. A classical deconvolution problem in the linear time-invariant system and signal framework is a special case of the general deconvolution problem, in which the underlying structure is the additive group. We broaden the classical framework to include a wide range of groups of both commutative and noncommutative type. These include finite, compact, and a large class of well-behaved locally compact groups that arise naturally in engineering applications. We developed a minimum mean-square solution for the deconvolution problem using the group representation theory and the concept of group stationarity.

There are many possible avenues for future research. At a theoretical level, one may extend the results of this study to blind deconvolution where the deblurring filter is unknown and has to be estimated along with the unknown signal from measurements. This task may necessitate the development of ARMA-type parametric models over groups. It will also be very interesting to perform deconvolution recursively within the Kalman filtering framework. At the application level, investigation of inverse filtering techniques for the Euclidean motion group and the affine group for image reconstruction problems as well as other applications are proceeding and will be reported in the future [76], [77].

\section{APPENDIX I}

Definitions of the basic concepts used in this paper are provided for readers' convenience. Detailed discussions and rigorous treatment of these concepts can be found in [69]-[71].

Definition A1.1:

- Let $G$ be a group, $F$ a field, and $V$ a vector field over $F$. A representation of a group $G$ is a homomorphism $\rho$ from $G$ into the group of automorphisms of $V$, denoted by $G L(V)$, i.e., $\rho: G \rightarrow G L(V)$, such that $\rho(g)=\rho_{g}$.

- If $V$ is an $n$-dimensional vector space over $F$, then for a fixed basis of $V$ there is an isomorphism $\varphi$ from $G L(V)$ into $G L(n, F)$. Therefore, $\varphi$ induces another representation $(\varphi \circ \rho)$ of $G$ into $G L(n, F)$, which is called a $m a-$ trix representation. Any representation of $G$ into $G L(V)$ is equivalent to a representation into $G L(n, F)$ and vice versa. The integer $n$ is called the degree of $\rho$.

\section{Definition A1.2:}

- Let $W$ be a subspace of $V$. If for all elements $g \in G, \rho_{g} v$ is again in $W\left(\rho_{g} W \subset W\right)$, then $W$ is said to be invariant under $\rho$ or, equivalently, $\rho$-invariant. If $V$ is nonempty and has no proper $\rho$-invariant subspace $W$ then the representation $\rho$ is said to be irreducible, else reducible.

- A group $G$ is called a topological group if $G$ is a topological space satisfying the Hausdorff separation axiom and the mapping $(x, y) \rightarrow x y^{-1}$ is a continuous mapping from $G \times G$ into $G$.

- Let $G$ be a topological group. A unitary representation of $G$ is a strongly continuous homomorphism $U$ from $G$ into the group of unitary operators of a Hilbert space $H, \boldsymbol{U}(H)$. $H$ is called the representation space of $U$ and denoted by $H(U)$. The dimension of $H(U)$ is the called the degree of $U$.

- Let $W$ be a subspace of a representation space $H(U)$ of a unitary representation $U$. Then $W$ is said to be invariant under $U$ if $U_{g} W \subset W$ for all $g \in G$. A unitary representation $U$ is called irreducible if $H(U)$ is nonempty and has no proper subspace invariant under $U$.

- Let $G$ be a locally compact topological group and let $H(R)=L^{2}(G)$ be the Hilbert space of square-integrable functions on $G$ with respect to right Haar measure on $G$. Let $f$ be a function in $H(R)$. Define the operator $R$ on $H(R)$ by $\lceil R(g) f\rceil(h)=f(h g)$. Then $R$ is a unitary representation of $G$ and is called the right regular representation of $G$. Similarly, the left regular representation $L$ is defined by $\lceil L(g) f\rceil(h)=f\left(g^{-1} h\right)$.

Definition A1.3:

- Let $G$ be a group, and let $K$ be a subgroup of $G$. Given an element $g \in G$, the subsets of the form

$$
g K=\{g k: k \in K\}, K g=\{k g: k \in K\}
$$

are called the left and right cosets of the subgroup $K$ determined by $g$. The equivalence class of the cosets are denoted by $G / K$ and $K \backslash G$, respectively.

- $G$ acts on the set $K \backslash G$ by right multiplications and since it is an automorphism of the group $K \backslash G$, it also induces automorphism over the representations of $K \backslash G$. Hence, this automorphism induces a representation of $G$ over the complex-valued function over $K \backslash G$ called the quasi-right regular representation.

\section{APPENDIX II}

\section{A. Proof of Theorem 1}

To simplify the notation, we shall denote $\mu(d t)$ by $d t$. Let

$$
\begin{gathered}
y(g)=\int_{X} L_{q}(g) f(t) x(t) d t, \\
\text { for some } f \in L^{2}(X, d t) \cap L(X, d t) \\
E\left[x\left(t_{1}\right) x\left(t_{2}\right)\right]=C_{x}\left(t_{1}, t_{2}\right), \text { for all } t_{1}, t_{2} \text { in } X \\
E\left[y\left(g_{1}\right) y\left(g_{2}\right)\right]=C_{y}\left(g_{1}, g_{2}\right), \text { for all } g_{1}, g_{2} \in G .
\end{gathered}
$$


Assume

$$
\begin{aligned}
E\left[x\left(g t_{1}\right) x\left(g t_{2}\right)\right]=E[ & \left.x\left(t_{1}\right) x\left(t_{2}\right)\right], \\
& \text { for all } t_{1}, t_{2} \text { in } X \text { and } g \text { in } G
\end{aligned}
$$

i.e.,

$$
C_{x}\left(t_{1}, t_{2}\right)=C_{x}\left(g t_{1}, g t_{2}\right) \text {, for all } t_{1}, t_{2} \text { in } X \text { and } g \text { in } G \text {. }
$$

Since $y(g), g \in G$ is a finite mean, finite variance process. Thus,

$$
\begin{aligned}
E[y & \left.\left(g_{1}\right) \overline{y\left(g_{2}\right)}\right] \\
& =C_{y}\left(g_{1}, g_{2}\right) \\
& =\int_{X} \int_{X} L_{q}\left(g_{1}\right) L_{q}\left(g_{2}\right) f\left(t_{1}\right) \overline{f\left(t_{2}\right)} C_{x}\left(t_{1}, t_{2}\right) d t_{1} d t_{2} \\
& =\int_{X} \int_{X} L_{q}\left(g_{1}\right) L_{q}\left(g_{2}\right) f\left(t_{1}\right) \overline{f\left(t_{2}\right)} C_{x}\left(g t_{1}, g t_{2}\right) d t_{1} d t_{2} .
\end{aligned}
$$

Note that the measure $d t$ is relatively invariant under left translations, i.e., $d g^{-1} t=\rho(g) d t$.

Let $s_{1}=g t_{1}$ and $s_{2}=g t_{2}$

$$
\begin{aligned}
= & \int_{X} \int_{X} L_{q}\left(g_{1}\right) L_{q}\left(g_{2}\right) f\left(g^{-1} s_{1}\right) \overline{f\left(g^{-1} s_{2}\right)} \\
& \cdot C_{x}\left(s_{1}, s_{2}\right) \rho_{\mu}^{2}(g) d s_{1} d s_{2} \\
= & \int_{X} \int_{X} L_{q}\left(g \circ g_{1}\right) L_{q}\left(g \circ g_{2}\right) f\left(s_{1}\right) \overline{f\left(s_{2}\right)} \\
& \cdot C_{x}\left(s_{1}, s_{2}\right) \rho_{\mu}^{2}(g) d s_{1} d s_{2} \\
= & \rho_{\mu}^{2}(g) C_{y}\left(g \circ g_{1}, g \circ g_{2}\right) .
\end{aligned}
$$

Thus, $y(g), g \in G$ is a left group stationary process. The proof of the "only if" part is similar and is omitted.

\section{B. Proof of Theorem 2}

The MMSE in (5.2a) is minimized when i.e.,

$$
E\left[\varepsilon_{W}(g) \overline{y(h)}\right]=0, \quad \text { for all } g, h \in G
$$

$$
E\left[\left(\int_{G} W(g, p) y(p) d p-x(g)\right) \overline{y(h)}\right]=0 .
$$

Assuming all processes involved have finite variance, (A2.1b) can be alternatively written as

$$
\int_{G} W(g, p) R_{y y}(p, h) d p=R_{x y}(g, h) .
$$

Under the assumptions that $x(g)$ and $y(g), g \in G$, are left group stationarity and jointly group stationary, i.e.,

$$
E\lceil x(g) \overline{y(h)}\rceil=R_{x y}\left(h^{-1} \circ g, e\right)
$$

(A2.1c) can be expressed as a group convolution

$$
\int_{G} W\left(p^{-1} \circ g, e\right) R_{y y}(p, e) d p=R_{x y}(g, e) .
$$

Furthermore, if $x(g)$ and $n(g), g \in G$, are statistically independent, then

$$
R_{x y}(g, e)=E[x(g) \overline{(x * f)(e)}] .
$$

It is straightforward to show that

$$
R_{x y}(g, e)=\left(\tilde{f} * R_{x}\right)(g)
$$

where $\tilde{f}(g)=\overline{f\left(g^{-1}\right)}$ and $R_{x}(g)=R_{x x}(g, e)$. On the other hand

$$
R_{y}(g)=\left(\tilde{f} * R_{x} * f\right)(g)+R_{n}(g)
$$

where $R_{y}(g)=R_{y y}(g, e)$ and $R_{n}(g)=R_{n n}(g, e)$. Thus, using (A2.3) and (A2.4), (A2.1d) can be written in Fourier domain as follows:

$$
\begin{array}{r}
\hat{W}_{\text {opt }}(\lambda)=S_{x}(\lambda) \hat{f}^{\dagger}(\lambda)\left[\hat{f}(\lambda) S_{x}(\lambda) \hat{f}^{\dagger}(\lambda)+S_{n}(\lambda)\right]^{-1}, \\
\lambda \in \hat{G} .
\end{array}
$$

Here, $\hat{f}^{\dagger}$ denotes the adjoint of the operator $\hat{f}, S_{x}$ and $S_{n}$ are the spectral density functions of $x(g)$ and $n(g)$, respectively. Note that the spectral density function is positive definite and hence its inverse exists.

The error covariance is given by

$$
\begin{aligned}
E[\varepsilon(g) \overline{\varepsilon(e)}] \\
=E[x(g) \overline{x(e)}]-E[x(g) \overline{(x * f * W)(e)}] \\
\quad+E[(n * W)(g) \overline{(n * W)(e)}]-E[(x * f * W)(g) \overline{x(e)}] \\
\quad+E[(x * f * W)(g) \overline{(x * f * W)(e)}] .
\end{aligned}
$$

In the Fourier domain, (A2.6a) becomes

$$
\begin{aligned}
& S_{\varepsilon}(\lambda)= \hat{W}(\lambda) S_{n}(\lambda) \hat{W}^{\dagger}(\lambda) \\
&+[I-\hat{W}(\lambda) \hat{f}(\lambda)] S_{x}(\lambda)[I-\hat{W}(\lambda) \hat{f}(\lambda)]^{\dagger}, \\
& \lambda \in \hat{G} .
\end{aligned}
$$

Thus, for the optimal filter given in (A2.5), the error power spectrum is given by

$$
S_{\varepsilon}(\lambda)=\left[I-\hat{W}_{\text {opt }}(\lambda) \hat{f}(\lambda)\right] S_{x}(\lambda), \quad \lambda \in \hat{G} .
$$

\section{ACKNOWLEDGMENT}

The author would like to thank the anonymous reviewers for comments, which helped improve the quality of the paper.

\section{REFERENCES}

[1] K. Kanatani, Group Theoretical Methods in Image Understanding. Berlin, Germany: Springer-Verlag, 1990.

[2] R. Lenz, Group Theoretical Methods in Image Processing (Lecture Notes in Computer Science). Berlin, Germany: Springer-Verlag, 1990.

[3] M. Ferraro, "Invariant pattern representations and lie group theory," in Advances in Electronics and Electron Physics. New York: Academic, 1992, vol. 84, pp. 131-196.

[4] R. J. Popplestone, "Group theory in robotics," in Proc. Robotics Research: The 1st Int. Symp., M. Brady and R. Paul, Eds. Cambridge, MA: MIT Press, 1984. 
[5] G. S. Chirikjian and I. Ebert-Uphoff, "Numerical convolution on the Euclidean group with applications to workspace generation," IEEE Trans. Robotics Automat., vol. 14, pp. 123-136, Feb. 1998.

[6] I. Ebert-Uphoff and G. S. Chirikjian, "Inverse kinematics of discretely actuated hyper redundant manupulators using workspace density," in Proc. IEEE Int. Conf. Robotics and Automation, Minneapolis, MN, 1996, pp. 139-145.

[7] R. E. Blahut, Algebraic Methods for Signal Processing and Communications Coding. New York: Springer-Verlag, 1991.

[8] W. Miller, "Topics in harmonic analysis with applications to radar and sonar," in Radar and Sonar, R. E. Blahut, W. Miller, and C. H. Wilcox, Eds. New York: Springer-Verlag, 1991, pp. 66-168.

[9] H. Naparst, "Dense target signal processing," IEEE Trans. Inform. Theory, vol. 37, pp. 317-327, Mar. 1991.

[10] S. Helgason, The Radon Transform, ser. Progress in Mathematics. Basel, Switzerland: Birkhauser, 1980, vol. 5.

[11] S. H. Izen, "A series inversion for the X-ray transform in $n$ dimensions," Inverse \Problems, vol. 4, pp. 725-748, 1988.

[12] S. R. Deans, The Radon Transform and Some of its Applications. New York: Wiley, 1983.

[13] C. E. Cook and M. Bernfeld, Radar Signals. New York: Academic, 1967.

[14] D. A. Swick, "A Review of Wideband Ambiguity Functions," Naval Research Laboratory, Washington, DC, NRL Rep. 6994, 1969.

[15] L. G. Weiss, "Wavelets and wideband correlation processing," IEEE Signal Processing Mag., vol. 11, no. 1, pp. 13-32, 1994.

[16] C. J. Nuzman and H. V. Poor, "Linear estimation of self-similar processes via Lamperti's transformation," J. Appl. Probab., vol. 37, no. 2, June 2000.

[17] H. L. Van Trees, Detection, Estimation, and Modulation Theory. New York: Wiley, 1977, vol. III.

[18] R. E. Blahut, "Theory of remote surveillance algorithms," in Radar and Sonar, Part I. New York: Springer Verlag, 1991, pp. 1-65.

[19] B. Yazici and R. L. Kashyap, "Second order stationary, self-similar models for $1 / f$ processes," IEEE Trans. Signal Processing, vol. 45, pp. 396-410, Feb. 1997.

[20] D. A. Pintsov, "Invariant pattern recognition, symmetry and the Radon transforms," J. Opt. Soc. Amer., vol. 6, no. 10, pp. 1545-1554, 1989.

[21] B. Yazici, "Group invariant methods in signal processing," in Proc. Conf. Information Sciences and Systems, Baltimore, MD, Mar. 1997 pp. $185-189$.

[22] B. Yazici and R. L. Kashyap, "Signal modeling and parameter estimation using scale stationary processes," in Proc. 1996 Int. Conf. Acoustics, Speech, and Signal Processing, vol. 5, Atlanta, GA, May 1996, pp. 2841-2844.

[23] — - "Affine stationary processes with applications to fractional Brownian motion," in Proc. 1997 Int. Conf. Acoustics, Speech, and Signal Processing, vol. 5, Munich, Germany, Apr. 1997, pp. 3669-3672.

[24] T. Inui, Y. Tanabe, and Y. Onodera, Group Theory and Its Applications in Physics. Berlin, Germany: Springer-Verlag, 1990.

[25] D. K. Maslen, "Fast transforms and sampling for compact groups," Ph.D. dissertation, Dept. Math., Harvard Univ., Cambridge, MA, 1993.

[26] D. H. Sattinger and O. L. Weaver, Lie Groups and Algebras with Applications to Physics, Geometry and Mechanics. New York: SpringerVerlag, 1986.

[27] J. R. Driscoll, D. Healy, and D. N. Rockmore, "Fast discrete polynomial transform with applications to data analysis for distance transitive graphs," SIAM J. Comput., vol. 26, pp. 1066-1099, 1997.

[28] D. N. Rockmore, "Efficient computation of Fourier inversion for finite groups," J. Assoc. Comput. Mach., vol. 41, no. 1, pp. 31-66, 1994.

[29] A. H. Doodley, "A nonabelian version of the Shannon sampling theorem," SIAM J. Math. Anal., vol. 20, no. 3, pp. 624-633, 1989.

[30] T. H. Glison, C. I. Black, and A. P. Sage, "On sonar signal analysis," IEEE Trans. Aerosp. Electron. Syst., vol. AES-6, pp. 37-49, Jan. 1970.

[31] A. Kak and M. Slaney, Principles of Computerized Tomography. Philadelphia, PA: SIAM, 2000.

[32] R. N. Bracewell and A. C. Riddle, "Inversion of fan beam scans in radio astronomy," J. Astrophys., vol. 24, pp. 2024-2033, 1967.

[33] L. S. Shepp and B. F. Logan, "The Fourier reconstruction of a head section," IEEE Trans. Nucl. Sci., vol. NS-21, pp. 21-43, June 1974.

[34] M. Holscheider, "Inverse Radon transforms through inverse wavelet transforms," Inverse Probl., vol. 7, pp. 853-861, 1991.

[35] P. Kuchment, K. Lancaster, and L. Mogilevskaya, "On local tomography," Inverse Probl., vol. 11, pp. 571-589, 1995.

[36] M. L. Brady, "A fast discrete approximation algorithm for the Radon transform," SIAM J. Comput., vol. 27, no. 1, pp. 107-119, 1998.
[37] O. F. Cools, G. C. Hermann, R. M. Van Der Weiden, and F. B. Kets, "Fast computation of the 3-D Radon transform," Geophysics, vol. 62, no. 1 , pp. 362-364, 1997.

[38] M. Defrise, R. Clack, and D. W. Townsend, "Image reconstruction from truncated, two dimensional, parallel projections," Inverse Probl., vol. 11, pp. 287-313, 1995.

[39] R. Ramamoorthi and P. Hanrahan, "A signal processing framework for inverse rendering," in Proc. 28th Annu. Conf. Computer Graphics and Interactive Techniques (SIGGRAPH), 2001, pp. 117-128.

[40] A. Robert, Introduction to the Representation Theory of Compact and Locally Compact Groups. Cambridge, U.K.: Cambridge Univ. Press, 1983.

[41] M. G. Karpovsky and E. A. Trachtenberg, "Some optimization problems for convolution systems over finite groups," Inform. Contr., vol. 34, pp. 227-247, 1977.

[42] E. A. Trachtenberg, "Systems over finite groups as suboptimal filters: A comparative study," in Proc. 5th Int. Symp. Mathematical Theory of Systems and Networks, Israel, June 1983, pp. 376-379.

[43] M. Karpovsky, Spectral Techniques and Fault Detection. New York: Academic, 1985.

[44] M. Artin, Algebra. Englewood Cliffs, NJ: Prentice-Hall, 1991.

[45] M. A. Naimark, Normed Rings. Groningen, The Netherlands: Noordhoff N.V., 1959.

[46] Harish- Chandra, "Representation of semi-simple Lie group on Banach space," Trans. Amer. Math. Soc., vol. 75, pp. 185-243, 1953

[47] J. Diximier, "Unitary representations of algebraic Lie groups," Ann. Inst. Fourier Gronoble, vol. 7, pp. 315-328, 1957.

[48] H. Reiter, Classical Harmonic Analysis and Locally Compact Groups, ser. Oxford Mathematical Monographs. Oxford, U.K.: Oxford Univ. Press, 1967.

[49] M. Duflo and C. C. Moore, "On the regular representation of a nonunimodular locally compact groups," J. Funct. Anal., vol. 21, pp. 209-243, 1976.

[50] A. M. Yaglom, "Second order homogeneous random fields," in Proc. 4th Berkley Symp. Mathematical Statistics and Probability. Berkeley, CA: Univ. Calif. Press, 1961, vol. 2, pp. 593-622.

[51] E. J. Hannan, Group Representations and Applied Probability, ser. Methuen's Review Series in Applied Probability. London, U.K.: Methuen \& Co., Ltd., 1965, vol. 3.

[52] P. Diaconis, Group Representations in Probability and Statistics. ser. Institute of Mathematical Statistics Monograph, S. S. Gupta, Ed. Hayward, CA: Inst. Math. Statist., 1988, vol. 11

[53] B. Mandelbrot and H. W. Van Ness, "Fractional Brownian motions, fractional noises and applications," SIAM Rev., vol. 10, pp. 422-436, 1968.

[54] A. N. Tikhonov and V. Y. Arsenin, Solutions of Ill-Posed Problems. New York: Wiley, 1977

[55] E. Parzen, "An approach to time series analysis," Annals Math. Statist., vol. 32, no. 4, pp. 951-989, Dec. 1961.

[56] A. B. Kyatkin and G. S. Chirikjian, "Regularized solution of a nonlinear convolution equation on the Euclidean group," Acta Applicandae Mathematicae, vol. 53, pp. 89-123, 1998 .

[57] A. Grossman, J. Morlet, and T. Paul, "Transforms associated to square integrable group representations-I: General results," J. Math. Phys., pp. 2473-2479, 1985

[58] H. Fuhr, "Sampling theorems for Heisenberg group," preprint, to be published.

[59] J. R. Higgins and R. L. Stens, Sampling Theory in Fourier and Signal Analysis. Advanced Topics. Oxford, U.K.: Oxford Univ. Press, 1999.

[60] A. B. Kyatkin and G. S. Chirikjian, "Pattern matching as a correlation on the discrete motion group," Comput. Vision Image Underst., vol. 74 pp. 22-35, Apr. 1999

[61] D. Bernier and K. F. Taylor, "Wavelets from square integrable representations," SIAM J. Math. Anal., vol. 27, no. 2, pp. 594-608, Mar. 1996.

[62] G. S. Chirikjian, "Conformational statistics of macromolecules using generalized convolution," Comput. Theor. Polymer Sci., vol. 11, pp. 143-153, Feb. 2001.

[63] P. T. Kim and J. Koo, "Optimal spherical deconvolution," J. Multivariate Anal., vol. 80, Jan. 2001

[64] R. Foote, G. Mirchandani, D. N. Rockmore, D. Healy, and T. Olson, "A wreath product group approach to signal and image processing. I Multiresolution analysis," IEEE Trans. Signal Processing, vol. 48, pp. 102-132, Jan. 2000

[65] G. Mirchandani, R. Foote, D. N. Rockmore, D. Healy, and T. Olson, "A wreath product group approach to signal and image processing. II. Convolution, correlation, and applications," IEEE Trans. Signal Processing, vol. 48, pp. 749-767, Mar. 2000 
[66] B. Yazici and M. Izzetoglu, "Optimal Wiener filtering for self-similar processes," in Proc of Int. Conf. Acoustics Speech and Signal Processing, vol. 2, 2002, pp. 1697-1700.

[67] B. Yazici, M. Izzetoglu, B. Onaral, and N. Bilgutay, "Kalman filtering for self-similar processes," in Proc. IEEE 11th Int. Workshop on Statistical Signal Processing, Aug. 2001, pp. 82-85.

[68] M. Izzetoglu, B. Onaral, N. M. Bilgutay, and P. R. Chitrapu, "Discrete time processing of linear scale invariant signals and systems," in Proc. SPIE Conf. Advanced Signal Processing Algorithms, Architectures, and Implementations X, Time-Frequency Analysis, vol. 4116, Aug. 2000.

[69] C. P. Milies and S. K. Sehgal, An Introduction to Group and Rings. Norwell, MA: Kluwer, 2002.

[70] L. C. Groove, Groups and Characters. New York: Wiley, 1997.

[71] A. L. Onishchik, Lie Groups and Lie Algebras I. New York: SpringerVerlag, 1993

[72] H. G. Feichtinger and C. Grochenig, "Coherent frames and irregular sampling," in Recent Advances in Fourier Analysis and its Applications (Lecture Notes in Mathematics), J Peetre et al., Ed. Berlin, Germany: Springer-Verlag, 1988, pp. 52-73.

[73] K. Gröchenig, "A new approach to irregular sampling of band-limited functions," in Recent Advances in Fourier Analysis and its Applications, J. S. and J. L. Byrnes, Ed. Norwell, MA: Kluwer, 1990, pp. 251-260.

[74] H. G. Feichtinger and K. Gröchenig, "Theory and practice of irregular sampling," in Wavelets: Mathematics and Applications, J. Benedetto and M. Frazier, Eds. Boca Raton, FL: CRC, 1993, pp. 305-363.
[75] A. Weil, L'Integration dans les Groupes Topologiques et ses Applications. Paris, France: Hermann, 1965.

[76] C. E. Yarman and B. Yazici, "Radon transform inversion via Wiener filtering over the Euclidean motion group," in Proc. Image Processing Conf. at the SPIE Medical Imaging 2003 Meeting, San Diego, CA, Feb. 2003.

[77] — " "Radon transform inversion via Wiener filtering over the Euclidean motion group," in Proc. IEEE Int. Conf. Image Processing, 2003, to be published.

[78] E. G. Kalnins and W. Miller, "A note on group contractions and radar ambiguity functions," in RADAR and SONAR, Part II. ser. IMA Volumes in Mathematics and its Applications, M. Bernfeld, R. Blahut, and A. Grunbaum, Eds. New York: Springer-Verlag, 1992.

[79] W. Schempp, Harmonic Analysis on the Heisenberg Nilpotent Lie Group, With Applications to Signal Theory. New York: Longman/ Wiley, 1986.

[80] W. Moran. Mathematics of radar signal processing. NATO Advanced Study Institute 2000 Meeting. [Online]. Available: http://www.cs.umb. edu/ asi/analysis2000/papers/moran.pdf

[81] M. I. Yadrenko, Spectral Theory of Random Fields, ser. Translation Series in Mathematics and Engineering. New York: Optimization Software Inc./Springer-Verlag, 1983.

[82] A. H. Tewfik, "Recursive estimation and spectral estimation for 2-d isotropic random fields," thesis, MIT, Cambridge, MA, 1987.

[83] N. Wiener, Cybernetics. Cambridge, MA: MIT Press, 1948. 